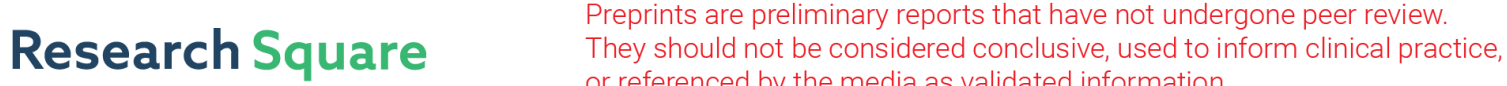 or referenced by the media as validated information. \\ De Broglie - Bohm Theorem in an underlying structure of Space and Spacetime
}

Salim Yasmineh ( $\square$ sayasmineh@gmail.com )

Brevalex https://orcid.org/0000-0002-9078-3306

Original Research

Keywords: simultaneity, non-locality, Hilbert space, configuration space, measurement, entanglement

Posted Date: February 9th, 2021

DOl: https://doi.org/10.21203/rs.3.rs-160859/v1

License: (9) This work is licensed under a Creative Commons Attribution 4.0 International License. Read Full License 
De Broglie - Bohm Theorem in an underlying structure of Space and Spacetime Salim YASMINEH

PhD University of Paris

Email: sayasmineh@gmail.com 


\title{
De Broglie - Bohm Theorem in an underlying structure of Space and Spacetime
}

\begin{abstract}
The concept of simultaneity is relative in special relativity whereas, it seems to have a definite meaning in quantum mechanics. On the other hand, theory and experiments in quantum mechanics have revealed the existence of non-local causal relations. We propose to use the invariant space-time interval introduced by special relativity as a benchmark for constructing a spacetime framework presenting a notion of an invariant time. We suggest that a physical system creates a non-local field within the spacetime framework and that the wavefunction of the physical system is an ontological entity that represents this non-local field. We propose to explain measurement and entanglement by using de Broglie Bohm theory in the context of the constructed spacetime framework.
\end{abstract}

Key words: simultaneity, non-locality, Hilbert space, configuration space, measurement, and entanglement.

\section{Introduction}

A quantum system for example made up of a pair of entangled particles behaves in such a manner that the quantum state of one particle cannot be described independently of the state of the other. This quantum phenomenon was first introduced as a thought experiment in the EPR paper [1] and it was later discovered that it can be experimentally testable by using Bell's inequality [2,3]. Standard quantum mechanics postulates that neither one of both particles has a determinate state until it is measured. As both particles are correlated, it is necessary that when the state of one particle is measured the second particle should 'simultaneously' acquire a determinate state. There have been numerous experiments such as Aspect's experiment [4] that proved the validity of quantum entanglement and hence of nonlocal causal connections. This phenomenon is discussed in detail by Jean Bricmont especially in relation to the de Broglie-Bohm theory [5,6] in his book "Making Sense of Quantum Mechanics" [7].

On the other hand, given that the laws of physics are invariant under Lorentz transformations, then there is no meaning of 'simultaneity' independently of any frame of reference and there should be no preferred frame of refence $[8,9]$. Thus, the notions of non-locality and simultaneity are inferred by quantum mechanics while being forbidden by special relativity.

We propose a solution to this dilemma by redefining the framework of spacetime in quantum mechanics without contradicting special relativity.

In section 2, we use the formalism of Minkowski spacetime to construct an underlying framework of spacetime referred to as 'phenomenal interface spacetime' related to standard spacetime by Lorentz transformations and presenting a notion of an invariant time. In sections 3-5, we introduce a global space structure composed of the 'phenomenal interface spacetime' in addition to an underlying space which shall be referred to as 'noumenal space' such that a particle in noumenal space creates a non-local field whose projection onto the phenomenal interface spacetime is represented by a wavefunction. From the standpoint of the phenomenal interface spacetime, if the particle is in noumenal space, the non-local field behaves like a wave whereas, when the particle is in the phenomenal interface spacetime, the particle behaves like a corpuscle. Measurement consists in 'bringing' a particle from noumenal space into the phenomenal interface spacetime. In sections 6-9 we define global space in the non-relativistic limit within which we consider de Broglie-Bohm theory. In addition to Schrodinger's equation and de Broglie Bohm's equation, we introduce a new relation that explains measurement and entanglement.

\section{Invariant spacetime structure}


We propose to use the formalism of special relativity to construct an invariant hyperbolic spacetime structure formed out of the foliation of a family of three-dimensional hyperboloids inside a light cone with respect to which the wavefunction will be defined.

\subsection{Review of Minkowski spacetime}

Hereafter, we shall use the formalism of Minkowski spacetime [10] as defined in a geometrical manner by Eric Gourgoulhon in his book 'Special Relativity in General frames' [11].

Minkowski spacetime $\mathcal{E}$ is defined as an affine space of dimension 4 on $\mathrm{R}$ endowed with a bilinear metric tensor $\mathrm{g}$ defined in an underlying vector space $\mathrm{E}$, of signature $(+,-,-,-)$. In the vector space $\mathrm{E}$, the set $\mathrm{C}$ composed of the zero vector and all null vectors form a light cone $\mathrm{C}$ composed of two sheets $\mathrm{C}^{+}$ and $\mathrm{C}^{-}$defining the future and past light cones, respectively.

Given the above defined spacetime $\mathcal{E}$ and given an arbitrary point $O \in \mathcal{E}$, a family of affine subspaces $\left(S_{\tau}\right)_{\tau \in R}$ is defined such that each subspace $S_{\tau}$ corresponds to the set of points of $\varepsilon$ that can be connected to $\mathrm{O}$ by a time-like vector $\overrightarrow{O M}$ of modulus $\tau$, where $\tau \in R$ :

$S_{\tau}=\left\{M \in \varepsilon, \overrightarrow{O M} \cdot \overrightarrow{O M}=-\tau^{2}<0\right\}$

We are henceforth, interested in physical systems that follow time-like or null worldlines and shall not consider the set of space-like vectors. In the spacetime $(\mathcal{E}, \mathrm{g})$, a point $M \in \mathcal{E}$ is said to belong to the subspace $S_{\tau}$ iff $\overrightarrow{O M} \cdot \overrightarrow{O M}=-\tau^{2}$. Each set of points $S_{\tau}$ is constituted of two subsets or two sheets $S_{\tau}^{+}$ and $S_{\tau}^{-}$:

$S_{\tau}^{+}=\left\{M \in S_{\tau}, \tau \geq 0\right\}$

$S_{\tau}^{-}=\left\{M \in S_{\tau}, \tau<0\right\}$

The positive sheets $S_{\tau}^{+}$and the negative sheets $S_{\tau}^{-}$belong to the interiors of the future $\mathrm{C}^{+}$and past $\mathrm{C}^{-}$ light cones, respectively.

Let $\left(x^{0}, x^{1}, x^{2}, x^{3}\right)$ be the coordinates of $M \in S_{\tau}$ in the affine frame defined by an origin $\mathrm{O}$ and a basis $\left(e_{\alpha}\right)$, then $\overrightarrow{O M} \cdot \overrightarrow{O M}=-\tau^{2}$ can be expressed as follows:

$-\left(x^{0}\right)^{2}+\left(x^{1}\right)^{2}+\left(x^{2}\right)^{2}+\left(x^{3}\right)^{2}=-\tau^{2}$

where $x^{o}=t, x^{1}=x / c, x^{2}=y / c, x^{3}=z / c$. Equation (2.4) can also be expressed as:

$\left(x^{0}\right)^{2}-\left(x^{1}\right)^{2}-\left(x^{2}\right)^{2}-\left(x^{3}\right)^{2}=\tau^{2}$

The above equation (2.5) is a three-dimensional hyperboloid of the two sheets $S_{\tau}^{+}$and $S_{\tau}^{-}$spanned by the free extremities of the time-like vectors $\overrightarrow{O M}$.

\subsection{Invariant hyperbolic spacetime}

We propose to consider the modulus $\tau$ of the time-like vector $\overrightarrow{O M}$ as an 'invariant time coordinate' for the family of affine subspaces $\left(S_{\tau}\right)_{\tau \in R}$ defined by (2.1). This family is constituted of three-dimensional hyperboloids where each hyperboloid is made up of two sheets $S_{\tau}^{+}$and $S_{\tau}^{-}$associated with the real coordinates $\tau \geq 0$ and $\tau<0$ respectively, and corresponding therefore, to a future-directed and a pastdirected invariant time $\tau$. The pile-up of this family of three-dimensional hyperboloids $\left(S_{\tau}\right)_{\tau \in R}$ inside the light cone $\mathrm{C}$ forms an invariant hyperbolic spacetime structure that we shall denote $\varepsilon_{I}$ where the index I refers to the term 'invariant'.

The two sheets $S_{\tau}^{+}$and $S_{\tau}^{-}$of each hyperboloid forms 'spatial-hypersurfaces' that we shall simply call 'slices' where each slice is associated with an invariant-time-coordinate $\tau \in R$. Thus, the invariant 
spacetime $\varepsilon_{I}$ is the foliation of the family $\left(S_{\tau}\right)_{\tau \in R}$ of three-dimensional hyperboloid slices $S_{\tau}$ at all the successive invariant instants $\tau$ :

$\varepsilon_{Q}=\cup_{\tau \in R} S_{\tau}=\cup_{\tau \in R}\left(S_{\tau}^{+} \cup S_{\tau}^{-}\right)$

All points on any given slice $S_{\tau}$ are associated with the same invariant instant $\tau$ which is indeed invariant to all observers from the perspective of any inertial frame of reference.

\subsection{Two-dimensional invariant hyperbolic spacetime}

Without any loss of generality, we will refer hereafter to a two-dimensional spacetime. Let $(0 ; x, t)$ be an orthonormal phenomenal frame of reference defined by the origin $\mathrm{O}$, a spatial $\mathrm{x}$-axis and a temporal $\mathrm{t}$-axis. We take $\mathrm{c}=1$, then the light cone is composed of the lines $\mathrm{X}$ and $\mathrm{Y}$ (where $\mathrm{X}$ is defined by $t=x$ and $\mathrm{Y}$ is defined by $t=-x)$ inside which are piled a family of simple hyperbolas $\left(S_{\tau}\right)_{\tau \in R}$. Let $(x, t)$ be the coordinates of a point $\mathrm{M}$ on a given hyperbola $\left(M \in S_{\tau}\right)$, then similarly to equation (2.5), $\overrightarrow{O M} \cdot \overrightarrow{O M}=-\tau^{2}$ can be expressed as follows:

$t^{2}-x^{2}=\tau^{2}$

For each invariant instant $\tau$, the free extremity $\mathrm{M}$ of the vector $\overrightarrow{O M}$ (of modulus $\tau$ ) spans the branches $S_{\tau}^{+}$and $S_{\tau}^{-}$of the hyperbolic-slice $S_{\tau}$. Hereafter, we shall only consider the positive branches $S_{\tau}^{+}$from which the upper index ' + ' shall be dropped to be simply denoted $S_{\tau}$.

The hyperbolic slice $S_{\tau}$ is a piecewise twice continuously differentiable curve of Minkowski spacetime $\left(\varepsilon_{I}, \mathrm{~g}\right)$ composed of a set of hyperbolic points $(u, \tau)$ where $\tau$ is considered to be an invariant time coordinate which is constant for each slice $S_{\tau}$ and $u$ is considered to be a hyperbolic space coordinate knowing that any vector tangent to $S_{\tau}$ is space-like.

The hyperbolic slice $S_{\tau}$ can be parametrized by a bijective function $\varphi$ from the points on the real axis $R$ into the points on that slice $S_{\tau}$ (i.e. $\varphi: R \rightarrow S_{\tau}$ ) such that any point $u$ on the slice $S_{\tau}$ is given by $u=$ $\varphi(\lambda) \equiv u(\lambda)$. The parameter $\lambda$ can be chosen to be the standard time coordinate $t$ or the standard space coordinate $x$.

The hyperbolic coordinates $(u, \tau)$ can also be defined with respect to the $\mathrm{X}$ and $\mathrm{Y}$ axes of the light cone. Indeed, let $(X, Y)$ be the coordinates of a point with respect to the cone's $\mathrm{X}$ and $\mathrm{Y}$ axes, then the corresponding hyperbolic coordinates $(u, \tau)$ are:

$u=\ln \sqrt{\frac{X}{Y}}$ and $\tau=\sqrt{2 X Y}$

The inverse mapping of the above equations (2.8) is:

$X=\frac{\tau e^{u}}{\sqrt{2}}$ and $Y=\frac{\tau e^{-u}}{\sqrt{2}}$

The spatial hyperbolic coordinate $u$ is constant when $Y=k X=e^{-2 u} X$ which is a straight line (or a ray $R_{u}$ ) passing through the origin. Thus, all points $(u, \tau)$ on the same ray $R_{u}$ share the same spatial hyperbolic coordinate $u$. The central ray $R_{0}$ (corresponding to an axe of symmetry with respect to the slices) represent the zero spatial coordinate (i.e $u=0$ ), while those on its right and left represent the positive $(u>0)$ and negative $(u<0)$ spatial hyperbolic coordinates respectively. On the other hand, all points $(u, \tau)$ on the same slice $S_{\tau}$ share the same invariant temporal coordinate $\tau$.

Thus, the rays $\left(R_{u}\right)_{u}$ and hyperbolic slices $\left(S_{\tau}\right)_{\tau}$ can be used to define a hyperbolic frame of reference $\left(O ; R_{u}, S_{\tau}\right)$ representing the invariant spacetime structure $\varepsilon_{I}$, where a given hyperbolic point $(u, \tau)$ is the intersection between the corresponding ray $R_{u}$ and slice $S_{\tau}$. 
On the other hand, the point $(X, Y)$ can be defined with respect to the orthonormal frame of reference $(O ; x, t)$ whose $x$ axis and $t$ axis are oriented at $\pm 45^{\circ}$ with respect to the $X$ axis or $Y$ axis. The relations between $(X, Y)$ and $(x, t)$ can thus, be expressed as follows:

$X=\frac{\sqrt{2}}{2}(t+x)$ and $\mathrm{Y}=\frac{\sqrt{2}}{2}(t-x)$

Therefore, by combining relations (2.9) and (2.10), the mapping between a point $(x, t)$ in the orthonormal frame of reference $(O ; x, t)$ and a hyperbolic point $(u, \tau)$ in the hyperbolic frame of reference $\left(O ; R_{u}, S_{\tau}\right)$ is:

$u=\ln \sqrt{\frac{t+x}{t-x}}$ and $\tau=\sqrt{t^{2}-x^{2}}$

The inverse mapping of (2.11) can be expressed as follows:

$x=\tau \sinh u$ and $t=\tau \cosh u$

On the other hand, by using expressions (2.12), the hyperbolic spatial coordinate $u$ can be expressed in function of invariant time $\tau$ as well as either one of the standard spatial coordinate $x$ and temporal coordinate $t$, as follows:

$u=\operatorname{arcosh}(t / \tau)=\ln \left(t / \tau+\sqrt{t^{2} / \tau^{2}-1}\right)$ where $t^{2} / \tau^{2} \geq 1$

$u=\operatorname{arcsinh}(x / \tau)=\ln \left(x / \tau+\sqrt{x^{2} / \tau^{2}+1}\right)$

Specifically, any given slice $S_{\tau}$ can be parametrized by the standard space coordinates $x$ according to expression (2.18), as follows:

$S_{\tau}=\left\{u_{\tau}(x) ; \tau\right.$ is constant, $x \in R$, and $\left.u_{\tau}(x)=\operatorname{arcsinh}(x / \tau)\right\}$

As each slice $S_{\tau}$ is associated with a corresponding invariant time $\tau$, then, all points $u$ belonging to that slice $S_{\tau}$ are said to be simultaneous. In other words, each slice $S_{\tau}$ is a class of simultaneity made up of a set of points $u_{\tau}$ that are associated to the same invariant instant $\tau$.

The passage from one slice $S_{\tau_{1}}$ into a subsequent slice $S_{\tau_{2}}$ represents the 'transition' of the invarianttime from one invariant instant $\tau_{1}$ to a consequent invariant instant $\tau_{2}$. The invariant time provides thus, an invariant time ordering of the set of slices. The invariant time increases as the slices $S_{\tau}$ depart from the origin. This invariant time ordering of the set of slices implies an invariant causality between points on different slices.

On the other hand, the mapping between a point $(u, \tau)$ in the hyperbolic frame of reference $\left(0 ; R_{u}, S_{\tau}\right)$ and a point $\left(x^{\prime}, t^{\prime}\right)$ in another inertial frame of reference $\left(O ; x^{\prime}, t^{\prime}\right)$ can be determined by simply using the Lorentz transformations.

Indeed, let $\left(O ; x^{\prime}, t^{\prime}\right)$ be another frame of reference that moves at a normalised constant velocity $\beta$ relative to $(0 ; x, t)$ where $\beta$ is a fraction of the speed of light $\mathrm{c}$ which is taken to be equal to 1 and hence, $\beta<1$. The Lorentz transformations are expressed as:

$t^{\prime}=\gamma(t-\beta x)$

$x^{\prime}=\gamma(x-\beta t)$

where $=\left(1-\beta^{2}\right)^{-1 / 2}$. Then by plugging (2.12) into the above equations, we get:

$x^{\prime}=\gamma \tau(\sinh u-\beta \cosh u)$ 
$t^{\prime}=\gamma \tau(\cosh u-\beta \sinh u)$

In view of the above, we postulate that the wavefunction (or state vector) is defined in a Hilbert space associated to the above constructed invariant hyperbolic spacetime structure. The arguments of the wavefunction are associated to the hyperbolic points $(u, \tau)$ of a corresponding slice $S_{\tau}$, and are thus, invariant with respect to time $\tau$. The wavefunction that we shall call 'invariant wavefunction' evolves according to the progression of subsequent slices. On the other hand, we will see in section 6 that the invariant wavefunction can be approximated by the standard wavefunction with respect to standard time and space coordinates.

\section{Hilbert Space}

In this section we propose to define the state vector of a particle and the structure of an underlying space.

\subsection{Invariant state vector}

Each slice $S_{\tau}$ can be associated to a Hilbert Space $\mathcal{H}$. The slice $S_{\tau}$ represents a position basis $\left\{\left|u_{\tau}\right\rangle\right\}$ of the associated Hilbert Space $\mathcal{H}$ with elements $\left|u_{\tau}\right\rangle$ labelled by a continuous hyperbolic position variable $u_{\tau}$ normalised using the Dirac $\delta$-function:

$\left\langle u_{\tau}^{\prime} \mid u_{\tau}\right\rangle=\delta\left(u_{\tau}^{\prime}-u_{\tau}\right)$

An invariant unit state vector $|\psi(\tau)\rangle$ in the Hilbert space $\mathcal{H}$ associated to the slice $S_{\tau}$ can then be expanded as an integral in function of the base elements $\left|u_{\tau}\right\rangle$ as follows:

$|\psi(\tau)\rangle=\int d u_{\tau} \psi\left(u_{\tau}, \tau\right)\left|u_{\tau}\right\rangle$

The right-hand side of the above equation is defined by a line integral along the piecewise smooth curve representing the slice $S_{\tau}$. It describes the state vector $|\psi(\tau)\rangle$ of a physical system as a superposition of position basis elements $\left|u_{\tau}\right\rangle$ each one of which corresponds to a definite hyperbolic position coordinate $u_{\tau}$ of the slice $S_{\tau}$ at a given invariant instant $\tau$. The expanding coefficients or 'weights' $\psi\left(u_{\tau}, \tau\right)$ represent a complex valued invariant wavefunction (i.e. defined with respect to an invariant instant $\tau$ ).

The left-hand side $|\psi(\tau)\rangle$ belongs to Hilbert space $\mathcal{H}$ and represents the resultant (i.e. the vector sum) of the decomposed position states. It seems reasonable to suppose that there should exist an elemental resultant position $\bar{u}$ in a real physical space which is associated to the resultant state vector $|\psi(\tau)\rangle$ belonging to Hilbert space $\mathcal{H}$. The elemental resultant position $\bar{u}$ would be the resultant or at least related to the position coordinates $u_{\tau}$ associated to the position basis elements $\left|u_{\tau}\right\rangle$.

If the resultant state vector $|\psi(\tau)\rangle$ is not reduced to a single basis element $\left|u_{\tau}\right\rangle$, then the elemental resultant position $\bar{u}$ cannot belong to the slice $S_{\tau}$ (i.e. $\bar{u} \notin S_{\tau}$ ). This implies that $\bar{u}$ cannot be referenced by the invariant temporal reference $\tau$. Indeed, as seen in section 2 , a point $M \in S_{\tau}$ if and only if $\overrightarrow{O M} \cdot \overrightarrow{O M}=-\tau^{2}$ and thus, if $\bar{u} \notin S_{\tau}$, then there exists no vector $\vec{V}$ representing $\bar{u}$ such that $\vec{V} \cdot \vec{V}=-\tau^{2}$. Therefore, the elemental resultant position $\bar{u}$ (if it exists and we assume it does) should belong to a timeless extension of space $\mathcal{N}$ such that:

$\bar{u} \in \mathcal{N}$ and $\mathcal{N} \cap S_{\tau}=\varnothing$

The temporal reference of the resultant state vector $|\psi(\tau)\rangle$ would then be emergent and would exist only from the standpoint of the hyperbolic slice $S_{\tau}$. It will be shown in section 7 that the resultant state vector $|\psi(\tau)\rangle$ represents a non-local field generated by a corresponding particle located at an elemental resultant position $\bar{u}$.

\subsection{Phenomenal Spacetime and Noumenal Space}


In view of the above, we postulate the existence of a four-dimensional space extension $\mathcal{N}$ whose all four dimensions are spatial. The ' $4 \mathrm{~d}$ space extension' $\mathcal{N}$ and each slice $S_{\tau}$ can thus be part of a higher $4 \mathrm{~d}$ structure that we shall call 'global space' $\mathcal{G}$. From the standpoint of each given slice $S_{\tau}$, the global space $\mathcal{G}$ structure may be thought of as being partitioned into three space blocks composed of the $3 \mathrm{~d}$ spatial part of the given slice $S_{\tau}$ acting as a boundary between two extradimensional $4 \mathrm{~d}$ spaces that compose the timeless spatial extension $\mathcal{N}$.

The pilling-up of the set of slices $\left\{S_{\tau}\right\}$ forms an 'interface spacetime' $\mathcal{J}\left(u_{\tau}, \tau\right)$ which is accessible through the window of standard spacetime $(x, t)$ to which it is related via the above formalism of Minkowski. However, the timeless space extension $\mathcal{N}$ corresponds to an underlying inaccessible space or rather not directly accessible. The standard $4 \mathrm{~d}$-spacetime as well as the directly related accessible interface spacetime shall be referred to as 'phenomenal standard spacetime' $P(x, t)$ and 'phenomenal interface spacetime' $\mathcal{J}\left(u_{\tau}, \tau\right)$ respectively, whereas the underlying non-directly accessible space extension $\mathcal{N}$ shall be referred to as 'noumenal space' $\mathcal{N}(\bar{u})$ borrowed from the 'noumenal and phenomenal worlds' of Kant.

In view of the above, the phenomenal interface spacetime (or simply 'interface spacetime') is defined as a set of interface spacetime points $\left(u_{\tau}, \tau\right)$, as follows:

$\mathcal{J}=\left\{\left(u_{\tau}, \tau\right) \in S_{\tau}: \tau \in R, u_{\tau} \in R,\left\{\left|u_{\tau}\right\rangle\right\}\right.$ is the base that spans Hilbert space $\left.\mathcal{H}\right\}$

The noumenal space $\mathcal{N}(\bar{u})$ is defined as the set of noumenal points $\bar{u}$, each being composed of an interface-like spatial coordinate $u$ and an extradimensional space coordinate $v$, as follows:

$\mathcal{N}=\{\bar{u}=(u, v): u, v \in R ; \forall|\psi(\tau)\rangle \in \mathcal{H}, \exists$ a corresponding elemental position $\bar{u} \in \mathcal{N}\}$

The global space $G$ is simply the union of the interface spacetime $\mathcal{J}\left(u_{\tau}, \tau\right)$ and noumenal space $\mathcal{N}(\bar{u})$ :

$\mathcal{G}=\mathcal{J} \cup \mathcal{N}$

To have a geometrical continuity in global space, the configuration in noumenal space should be similar to the slicing structure in interface spacetime. Thus, noumenal space $\mathcal{N}$ can be considered as an affine space partitioned into a family of affine subspaces $\left(S_{v}\right)_{v \in R}$ congruent to each interface slice $S_{\tau}$. The family of affine subspaces $\left(S_{v}\right)_{v \in R}$ is constituted of a family of three-dimensional hyperboloids where each hyperboloid is made up of two sheets $S_{v}^{+}$and $S_{v}^{-}$associated with the real extradimensional space coordinates $v \geq 0$ and $v<0$ respectively corresponding to positive and negative invariant coordinates $v$. The piling-up of this family of three-dimensional hyperboloids $\left(S_{v}\right)_{v \in R}$ forms an invariant hyperbolic space structure of the $4 \mathrm{~d}$ noumenal space. The two sheets $S_{v}^{+}$and $S_{v}^{-}$of each hyperboloid forms 'noumenal slices' where each slice is associated with an invariant extradimensional space coordinate $v \in R$. The noumenal space is a foliation of the family $\left(S_{v}\right)_{v \in R}$ of three-dimensional hyperboloids $S_{v}$ at all invariant space coordinates $v$, such that, given any arbitrary interface slice $S_{\tau}$, there exists a set of geometrically congruent noumenal slices $\left\{S_{v}\right\}$. In noumenal space the extradimensional space coordinate $v$ is a spatial invariance that plays a role similar to the temporal invariance coordinate $\tau$ of the interface spacetime.

Thus, by introducing the invariance of the extradimensional space coordinate $v$, expression (3.5) becomes:

$\mathcal{N}=\left\{\bar{u}=\left(u_{v}, v\right) \in S_{v}: u_{v}, v \in R ; \forall|\psi(\tau)\rangle \in \mathcal{H}, \exists\right.$ corresponding position $\left.\left(u_{v}, v\right) \in S_{v}\right\}$

In view of expressions (3.5), (3.6) and (3.7), the global space $\mathcal{G}$ can be defined as a set of global points $\tilde{u}$ composed of hyperbolic spatial coordinates $u_{\gamma}$ and invariant coordinates $\gamma$, as follows:

$\mathcal{G}=\left\{\tilde{u}=\left(u_{\gamma}, \gamma\right): u_{\gamma}, \gamma \in R ;\right.$ for $\gamma=\tau, \tilde{u} \in S_{\tau}$, and for $\left.\gamma=v, \tilde{u} \in S_{v}\right\}$ 


\section{Noumenal Space}

The fact that noumenal space is non-temporal does not imply that a particle in this space cannot have different positions. It entails however, that the different positions have no temporal ordering. But, as the extradimensional space coordinate $v$ is invariant for each slice, then all changes between points on different slices can be defined with respect to changes in the extradimensional coordinate $v$. Even though, the invariant extradimensional space coordinate $v$ has no specific direction, yet different positions of a particle in noumenal space can still be characterised by an undirected ordering relation of the invariant coordinate $v$. This undirected ordering can be defined by a 'betweenness relation' as in the C-relations identified by McTaggart [12] and as explained by Baron and Miller [13]. Indeed, a 'betweenness relation' defines an unchanging fact that a particle at an invariant coordinate $v_{B}$ in noumenal space can be considered as between its $v_{A}$ and $v_{C}$ extradimensional space coordinates without appealing to any ordering. The relation ' $v_{B}$ between $v_{A}$ and $v_{C}$ ' is an unchanging fact in noumenal space but is undirected in the sense that there is no fact of matter as to the direction of the sequence of positions.

Thus, a change in noumenal space has a sense and can conceptually be described by means of an undirected ordering relation. On the other hand, due to the lack of any temporal notion, spatially separated objects in noumenal space can be non-locally interconnected behaving as a single 'holistic identity'. Such a holistic identity can for example be composed of a group of spatially separated particles whose features form a single state at a given invariant coordinate $v$. In other words, all parts of a holistic identity should have the same invariant coordinate $v$. Indeed, the invariant spatial coordinate $v$ in noumenal space plays a similar role to that of the invariant time coordinate $\tau$ in interface spacetime such that a relation of events happening at the same invariant time coordinate $\tau$ (i.e. simultaneity in interface spacetime) corresponds to a relation of states taking place at the same invariant space coordinate $v$ in noumenal space.

\section{Relation between Noumenal Space and Phenomenal Interface Spacetime}

In this section we propose to treat very schematically and in a conceptual manner, the relation between noumenal space and interface spacetime and specifically, the indirect access to the former through its potential effects on the latter.

\subsection{Noumenal particle and non-local noumenal field}

Starting from the matter of fact that theory and experiments in quantum mechanics entail non-local causal connections, we postulate the existence of a non-local noumenal field $\Phi$ created by each physical system in noumenal space. The non-local noumenal field $\Phi$ depends mainly on the position and movement of the system in noumenal space as well as other physical features of the system. Hereafter, a system, or a particle located in noumenal space shall be called 'noumenal system' or 'noumenal particle'.

Postulate 1: The state of a noumenal particle (i.e. a particle in noumenal space) can be indirectly specified by an invariant wavefunction $\psi_{\bar{U}}\left(u_{\tau}, \tau\right)$ representing the density distribution on the interface slice $S_{\tau}$ of a non-local projected noumenal field $\varphi_{\bar{U}}\left(u_{\tau}, \tau\right)$ created by the noumenal particle.

First, we postulate that a noumenal particle located at a noumenal position $\bar{U}=(U, v)$ creates a nonlocal noumenal field $\Phi_{\bar{U}}$ at all global points $\left\{\left(u_{\gamma}, \gamma\right)\right\}$. The non-local noumenal field can be expressed as a function $\Phi_{\bar{U}}$ mapping the set of global points $\left\{\left(u_{\gamma}, \gamma\right)\right\}$ into a set of values $\left\{\Phi_{\bar{U}}\left(u_{\gamma}, \gamma\right)\right\}$ characterising the non-local noumenal field effect at each global point: 


$$
\left\{\left(u_{\gamma}, \gamma\right)\right\} \stackrel{\Phi_{\bar{U}}}{\longrightarrow}\left\{\Phi_{\bar{U}}\left(u_{\gamma}, \gamma\right)\right\}
$$

The non-local noumenal field $\Phi_{\bar{U}}\left(u_{\gamma}, \gamma\right)$ at a global point $\left(u_{\gamma}, \gamma\right)$ depends on the coordinates of that point as well as the noumenal position $\bar{U}$, the latter dependence being indicated by a subindex attached to the non-local noumenal field $\Phi_{\bar{U}}$. The noumenal position $\bar{U}$ is in fact related to the set of noumenal field values $\left\{\Phi_{\bar{U}}\left(u_{\gamma}, \gamma\right)\right\}$ by a two-way relation:

$$
\bar{U} \leftrightarrow\left\{\Phi_{\bar{U}}\left(u_{\gamma}, \gamma\right)\right\}
$$

Any variation $\delta \Phi_{\bar{U}}\left(u_{\gamma}, \gamma\right)$ exacted on the noumenal field at a global point $\left(u_{\gamma}, \gamma\right)$ generates a variation in the noumenal position $\delta \bar{U}$ which in its turn creates a variation of the noumenal field at all other points $\left\{\Phi_{\delta \bar{U}}\left(u_{\gamma}, \gamma\right)\right\}$. In principle, any point in global space may non-locally affect any other point via the timeless noumenal space by passing through the intermediate noumenal physical system.

According to expression (3.8), a global point $\left(u_{\gamma}, \gamma\right)$ can be either an interface point $\left(u_{\tau}, \tau\right)$ belonging to an interface slice $S_{\tau}$ or a noumenal point $\left(u_{v}, v\right)$ belonging to a noumenal slice $S_{v}$ :

$$
\left(u_{\gamma}, \gamma\right)= \begin{cases}\left(u_{\tau}, \tau\right) \in S_{\tau} & \text { interface point } \\ \left(u_{v}, v\right) \in S_{v} & \text { noumenal point }\end{cases}
$$

Our main interest here is the part of the noumenal field which we shall call 'projected non-local noumenal field' noted $\varphi_{\bar{U}}$, that affects the phenomenal interface slice $S_{\tau}$ and that maps the set of interface points $\left\{\left(u_{\tau}, \tau\right) \in S_{\tau}\right\}$ into a set of values $\left\{\varphi_{\bar{U}}\left(u_{\tau}, \tau\right)\right\}$ characterising the projected non-local noumenal field effect at each interface point:

$$
\left\{\left(u_{\tau}, \tau\right)\right\} \stackrel{\varphi_{\bar{U}}}{\longrightarrow}\left\{\varphi_{\bar{U}}\left(u_{\tau}, \tau\right)\right\}
$$

The value of the non-local noumenal field $\varphi_{\bar{U}}\left(u_{\tau}, \tau\right)$ at any interface point $\left(u_{\tau}, \tau\right)$ depends mainly on the position $\bar{U}=(U, v)$ of the noumenal particle with respect to the interface point $\left(u_{\tau}, \tau\right)$ which can be represented by a vector $a\left(u_{\tau}, \tau, \bar{U}\right) \equiv a_{\bar{U}}\left(u_{\tau}, \tau\right)$ between these two points. This vector $a_{\bar{U}}\left(u_{\tau}, \tau\right)$ may be defined by its orientation $\theta_{\bar{U}}\left(u_{\tau}, \tau\right)$ and its module $\left\|a_{\bar{U}}\left(u_{\tau}, \tau\right)\right\|$, the latter being the distance $d\left(\mathrm{u}_{\tau}-\bar{U}\right)$ between the interface spatial coordinate $\mathrm{u}_{\tau}$ and the noumenal position $\bar{U}$. The vector $a_{\bar{U}}\left(u_{\tau}, \tau\right)$ can be expressed by a complex number, as follows:

$a_{\bar{U}}\left(u_{\tau}, \tau\right)=\left\|a_{\bar{U}}\left(u_{\tau}, \tau\right)\right\| e^{i \theta_{\bar{U}}\left(u_{\tau}, \tau\right)}=\left(d\left(\mathrm{u}_{\tau}-\bar{U}\right)\right) e^{i \theta_{\bar{U}}\left(u_{\tau}, \tau\right)}$

The noumenal position $\bar{U}$ has no temporal reference. Thus, we introduce the notion of a 'projected position' $U(\tau)$ of the noumenal position $\bar{U}$ on the interface slice $S_{\tau}$. The projected position $U(\tau)$ is referenced by the invariant time $\tau$ associated to the interface slice $S_{\tau}$ where it subsists as a 'shadowposition' of the corresponding noumenal position $\bar{U}$ that exists in noumenal space $\mathcal{N}$.

From the standpoint of any interface point $\left(u_{\tau}, \tau\right)$, the noumenal position $\bar{U}$ (i.e. its module and orientation) can be expressed in function of the projected position $U(\tau)$ and the extradimensional space coordinate $v$. For example, the distance $d\left(\mathrm{u}_{\tau}-\bar{U}\right)$ between an interface spatial coordinate $\mathrm{u}_{\tau}$ and the noumenal position $\bar{U}$ can be defined in function of a first distance between the interface point $u_{\tau}$ and the projected position $U(\tau)$ and a second distance corresponding to the value of the extradimensional space coordinate $v$, as follows:

$d\left(\mathrm{u}_{\tau}-\bar{U}\right)=\left\|a_{\bar{U}}\left(u_{\tau}, \tau\right)\right\|=\sqrt{\left(d\left(\mathrm{u}_{\tau}-U(\tau)\right)\right)^{2}+v^{2}}$

Therefore, the vector $a_{\bar{U}}\left(u_{\tau}, \tau\right)$ can be expressed in function of the interface point $\left(u_{\tau}, \tau\right)$, the projected position $U(\tau)$, and the extradimensional space coordinate $v$. However, for simplicity we will continue 
to use $\bar{U}$ as a subindex for noting the vector $a_{\bar{U}}$ by keeping in mind that it can also be expressed in function of $U(\tau)$ and $v$.

The density magnitude (or module) of the non-local noumenal field $\varphi_{\bar{U}}\left(u_{\tau}, \tau\right)$ decreases as the distance $d\left(\mathrm{u}_{\tau}-\bar{U}\right)$ between the interface space coordinate $\mathrm{u}_{\tau}$ and the noumenal position $\bar{U}$ increases. In fact, by supposing the noumenal field to be composed of homogeneous and isotropic field lines stemming out from a noumenal particle, it is geometrically trivial that the density of field lines (i.e. number of field lines per unit area) decreases as the distance between the noumenal particle and a point at the interface spacetime increases. The density magnitude of the non-local projected noumenal field $\varphi_{\bar{U}}\left(u_{\tau}, \tau\right)$ can thus be defined by the module $\left\|g\left(a_{\bar{U}}\left(u_{\tau}, \tau\right)\right)\right\|$ of a normalised complex-valued function $g\left(a_{\bar{U}}\left(u_{\tau}, \tau\right)\right)$ such that the module $\left\|g\left(a_{\bar{U}}\left(u_{\tau}, \tau\right)\right)\right\|$ is a decreasing function with respect to the module $\left\|a\left(u_{\tau}, \tau\right)\right\|$ of the complex number $a_{\bar{U}}\left(u_{\tau}, \tau\right)$ :

$$
\begin{aligned}
& \left\|a_{\bar{U}}\left(u_{\tau}, \tau\right)\right\|<\left\|a_{\bar{U}}\left(u_{\tau}^{\prime}, \tau\right)\right\| \rightarrow\left\|g\left(a_{\bar{U}}\left(u_{\tau}, \tau\right)\right)\right\|>\left\|g\left(a_{\bar{U}}\left(u_{\tau}^{\prime}, \tau\right)\right)\right\| \\
& \int\left\|g\left(a_{\bar{U}}\left(u_{\tau}, \tau\right)\right)\right\|^{2} d \mathrm{u}_{\tau}=1
\end{aligned}
$$

The non-local projected noumenal field $\varphi_{\bar{U}}\left(u_{\tau}, \tau\right)$ represents thus a complex-valued density distribution within the phenomenal interface slice $S_{\tau}$ and can be expressed in polar form, as follows:

$$
\varphi_{\bar{U}}\left(u_{\tau}, \tau\right)=g\left(a_{\bar{U}}\left(u_{\tau}, \tau\right)\right)=g\left(\left(d\left(u_{\tau}-\bar{U}\right)\right) e^{i \theta\left(u_{\tau}, \tau\right)}\right)=A_{\bar{U}}\left(u_{\tau}, \tau\right) e^{i F_{\bar{U}}\left(u_{\tau}, \tau\right)}
$$

\subsection{Invariant wavefunction and projected non-local noumenal field}

As expressed in equation (3.1), each phenomenal interface slice $S_{\tau}$ represents a position basis of an associated Hilbert Space $\mathcal{H}$. It can then, be reasonably suggested that the non-local projected noumenal field $\varphi_{\bar{U}}\left(u_{\tau}, \tau\right)$ within the slice $S_{\tau}$ is represented in the associated Hilbert space by a complex-valued invariant wavefunction $\psi_{\bar{U}}\left(u_{\tau}, \tau\right)$. The amplitude $R_{\bar{U}}\left(u_{\tau}, \tau\right)$ and phase $S_{\bar{U}}\left(u_{\tau}, \tau\right)$ of the invariant wavefunction $\psi_{\bar{U}}\left(u_{\tau}, \tau\right)$ is related to the amplitude $A_{\bar{U}}\left(u_{\tau}, \tau\right)$ and phase $F_{\bar{U}}\left(u_{\tau}, \tau\right)$ of the projected noumenal field $\varphi_{\bar{U}}\left(u_{\tau}, \tau\right)$ :

$\psi_{\bar{U}}\left(u_{\tau}, \tau\right)=R_{\bar{U}}\left(u_{\tau}, \tau\right) e^{i S_{\bar{U}}\left(u_{\tau}, \tau\right)} \leftrightarrow \varphi_{\bar{U}}\left(u_{\tau}, \tau\right)$

The invariant wavefunction $\psi_{\bar{U}}\left(u_{\tau}, \tau\right)$ or each one of the amplitude $R_{\bar{U}}\left(u_{\tau}, \tau\right)$ and phase $S_{\bar{U}}\left(u_{\tau}, \tau\right)$, depends on the interface point $\left(u_{\tau}, \tau\right)$ and the noumenal position $\bar{U}$ (or the projected position $U(\tau)$, and the extradimensional space coordinate $v$ instead of $\bar{U}$ ). Specifically, expression (5.9) indicates that the amplitude $R_{\bar{U}}\left(u_{\tau}, \tau\right)$ is related to the distance $d\left(\mathrm{u}_{\tau}-\bar{U}\right)$ between the interface point $\mathrm{u}_{\tau}$ and the noumenal position $\bar{U}$ while the phase $S_{\bar{U}}\left(u_{\tau}, \tau\right)$ is related to the direction $\theta_{\bar{U}}\left(u_{\tau}, \tau\right)$ of the straight line joining the interface point $\mathrm{u}_{\tau}$ to the noumenal particle.

Each argument $\left(u_{\tau}, \tau\right)$ of the invariant wavefunction $\psi_{\bar{U}}\left(u_{\tau}, \tau\right)$ represents a 'shadow-position' associated with a 'shadow-momentum' of the noumenal particle that has a real position $\bar{U}$ in noumenal space noting however, that there is no sense in associating a real momentum to the noumenal particle in a timeless noumenal space. All the arguments of the invariant wavefunction $\psi_{\bar{U}}\left(u_{\tau}, \tau\right)$ are thus, shadowpositions whose median is represented by the projected position $U(\tau)$. These shadow-positions and associated shadow-momenta can be considered as the positions and momenta of a 'fictious-particle' (or 'ghost-particle') omnipresent all over the interface slice $S_{\tau}$. Nevertheless, all these shadow-positions of the phenomenal interface slice $S_{\tau}$ are representatives of a real unique position $\bar{U}$ in noumenal space.

\subsection{Implicit variables of the invariant wavefunction}

The unique noumenal position $\bar{U}$ (or equivalently, the couple $(U(\tau), v)$ ) is a hidden 'non-local' variable or at least a non-explicit variable of the invariant wavefunction $\psi_{\bar{U}}\left(u_{\tau}, \tau\right)$. It is in fact implicitly defined in the expression of the wavefunction: the interface point at which the amplitude $R_{\bar{U}}\left(u_{\tau}, \tau\right)$ has its 
maximum value indicates the projected position $U(\tau)$ while the dispersion of the amplitude $R_{\bar{U}}\left(u_{\tau}, \tau\right)$ is related to the magnitude of the interdimensional coordinate $v$.

Indeed, according to equation (5.6), the distance $d(U(\tau)-\bar{U})$ between the projected position $U(\tau)$ and the noumenal position $\bar{U}$ is equal to the extradimensional space coordinate $v$ and represents the minimal distance from the noumenal particle knowing that by definition, the extradimensional space coordinate $v$ is equal to zero at the interface slice $S_{\tau}$. On the other hand, as the amplitude $R_{\bar{U}}\left(u_{\tau}, \tau\right)$ is related to the distance $d\left(\mathrm{u}_{\tau}-\bar{U}\right)$ between the interface point $\mathrm{u}_{\tau}$ and the noumenal position $\bar{U}$ and as this distance is minimal at the projected position $U(\tau)$ then, the amplitude $R_{\bar{U}}\left(u_{\tau}, \tau\right)$ should have its maximum value at the projected position $U(\tau)$ and should decrease as we move further away from $U(\tau)$. For example, the amplitude may have the form of a Gaussian distribution centered at the projected position $U(\tau)$. On the other hand, if the interdimensional coordinate $v$ tends to zero, the noumenal position $\bar{U}$ of the noumenal particle tends towards the latest projected position $U(\tau)$ and thus, the amplitude becomes more and more peaked around the projected position. This suggests that the interdimensional coordinate $v$ should be implicitly related to the dispersion $\sigma$ of the amplitude's distribution.

\subsection{Indirect noumenal state of a noumenal particle}

The noumenal position $\bar{U}$ as well as the non-local connections are thus, inherent in the invariant wavefunction $\psi_{\bar{U}}\left(u_{\tau}, \tau\right)$. Nevertheless, to have a complete picture of quantum phenomena especially, the non-local effects, it is necessary to consider also the noumenal position $\bar{U}$ (or equivalently, the projected position $U(\tau)$ and the extradimensional space coordinate $v$ ) in the formulation of quantum rules.

Proposition 1: From the perspective of the interface spacetime, the features of a noumenal particle can be specified by an indirect noumenal state $\Omega_{\bar{U}}(\tau)$ composed of the invariant wavefunction $\psi_{\bar{U}}\left(u_{\tau}, \tau\right)$ in association to the projected position $U(\tau)$ on the interface slice $S_{\tau}$ at an invariant instant $\tau$ as well as the corresponding extradimensional space coordinate $v$ :

$$
\Omega_{\bar{U}}(\tau)=\left(\psi_{\bar{U}}\left(u_{\tau}, \tau\right), U(\tau), v, \tau\right)
$$

As in expression (5.2), any variation in the noumenal position $\delta \bar{U}$ of a noumenal particle generates a variation in the noumenal field $\delta \Phi_{\bar{U}}$ entailing a variation on its projected field $\delta \varphi_{\bar{U}}\left(u_{\tau}, \tau\right)$ and thus, a variation of the invariant wavefunction $\delta \psi\left(\mathrm{u}_{\tau}, \tau\right)$ :

$$
\delta \bar{U} \leftrightarrow \delta \varphi_{\bar{U}}\left(u_{\tau}, \tau\right) \leftrightarrow \delta \psi_{\bar{U}}\left(u_{\tau}, \tau\right) \leftrightarrow \delta\left(R_{\bar{U}}\left(u_{\tau}, \tau\right), S_{\bar{U}}\left(u_{\tau}, \tau\right)\right)
$$

The above relations (5.12) define an action-reaction process between the state of the noumenal particle and the invariant wavefunction. These relations should be formulated from the standpoint of the phenomenal interface spacetime into one or more rules.

It should be noted however, that any non-local causal connection between two points in the interface slice $S_{\tau}$ is not conveyed within the interface slice $S_{\tau}$ and can only take place in noumenal space via the non-local noumenal field $\varphi_{\bar{U}}$ represented by the invariant wavefunction $\psi(\tau, u)$, by passing through the corresponding intermediate noumenal particle.

\subsection{Rules related to the indirect noumenal state}

It is reasonable to suppose that a first rule should be a smooth evolution of the invariant wavefunction $\psi_{\bar{U}}\left(u_{\tau}, \tau\right)$, i.e., a transformation infinitely close to identity equivalent to Schrodinger equation. This first rule would govern the evolution of the invariant wavefunction $\psi_{\bar{U}}\left(u_{\tau}, \tau\right)$ representing the non-local projected noumenal field on an interface slice $S_{\tau}$ into a consequent interface wavefunction $\psi_{\bar{U}}\left(u_{\tau}, \tau+\delta \tau\right)$ representing the projected noumenal field on the subsequent interface slice $S_{\tau+\delta \tau}$ according to an infinitesimal unitary transformation $\mathcal{U}(\delta \tau)$, as follows: 
$\psi_{\bar{U}}\left(u_{\tau}, \tau+\delta \tau\right)=\mathcal{U}(\delta \tau) \psi_{\bar{U}}\left(u_{\tau}, \tau\right)$

A second rule could be a relation between the projected position $U(\tau)$ and the phase $S_{\bar{U}}\left(u_{\tau}, \tau\right)$ of the invariant wavefunction $\psi_{\bar{U}}\left(u_{\tau}, \tau\right)$ similar to the de Broglie Bohm law. Indeed, a variation in the direction $\theta_{\bar{U}}\left(u_{\tau}, \tau\right)$ of the straight line joining the interface point $\mathrm{u}_{\tau}$ to the noumenal particle implies a variation in phase $S_{\bar{U}}\left(u_{\tau}, \tau\right)$ which necessarily implies a variation of the first component $U$ of the noumenal position $\bar{U}=(U, v)$ and consequently, a variation of the projected position $U(\tau)$ and this last variation can be assimilated to the momentum $p(\tau)$ of a fictitious particle at the current projected position $U(\tau)$ :

$\delta S\left(u_{\tau}, \tau\right) \leftrightarrow \delta U(\tau) \leftrightarrow p(\tau)$

A third rule should then concern the invariant interdimensional coordinate $v$. A variation in $v$ does not necessarily imply a variation in the projected position $U(\tau)$ though, it necessarily implies a variation in the distance of the noumenal particle from the interface slice $S_{\tau}$ which causes a variation in the amplitude $R_{\bar{U}}\left(u_{\tau}, \tau\right)$ of the invariant wavefunction $\psi_{\bar{U}}\left(u_{\tau}, \tau\right)$ :

$\delta R\left(u_{\tau}, \tau\right) \leftrightarrow \delta v$

\subsection{Measurement}

The projected position may be considered to evolve according to a sequence $(U(\tau))_{n(\tau)}$ of projected positions matching a corresponding evolution in the first component $U$ of the noumenal position $\bar{U}=$ $(U, v)$ according to a corresponding sequence of noumenal positions $(\bar{U})_{n}=\left((U)_{n},(v)_{n}\right)$. Any variation in noumenal position $\Delta \bar{U}$ and specifically, in its first component $\Delta U$ is timeless while its corresponding variation in the projected position $\Delta U(\tau)$ takes place during a certain time period $\Delta \tau$ and thus, the sequence of projected positions $(U(\tau))_{n(\tau)}$ depends on the invariant time $\tau$. At any invariant instant $\tau$, each projected position $U_{n(\tau)}(\tau)$ represents the point in the interface slice $S_{\tau}$ having the minimal distance from the noumenal particle which is equal to $v_{n}$ and thus, when the invariant coordinate $v_{n}$ tends to zero, the noumenal position $\bar{U}_{n}$ tends to the latest projected position $U_{L}(\tau)$ :

$\forall \varepsilon>0, \exists \delta>0: \forall v_{n},\left|v_{n}-0\right|<\delta \rightarrow\left|\bar{U}_{n}-U_{L}(\tau)\right|<\varepsilon$

Finally, when $v_{n}$ becomes equal to zero, the noumenal position $\left(U_{n}, v_{n}\right)$ becomes congruent to the latest projected position $U_{L}(\tau)$ which in fact becomes the real interface position of the particle, as indicated in the following proposition.

Proposition 2: Measurement is a local interaction with the projected noumenal field $\varphi_{\bar{U}}\left(u_{\tau}, \tau\right)$ which in its turn acts non-locally on the corresponding noumenal particle 'bringing' it from noumenal space into the phenomenal interface slice $S_{\tau}$ at a definite position corresponding to the latest projected position $U_{L}(\tau)$. Mathematically, it can be defined by an operation $\mathrm{M}$ acting on the invariant wavefunction $\psi_{\bar{U}}\left(u_{\tau}, \tau\right)$ and consisting in taking the limit of the latter as the interdimensional coordinate $v$ tends to zero, the limit being the latest projected position $U_{L}(\tau)$ :

$M \psi_{\bar{U}}\left(u_{\tau}, \tau\right)=\lim _{v \rightarrow 0} \psi_{\bar{U}}\left(u_{\tau}, \tau\right)=U_{L}(\tau)$

Measurement can also be defined with respect to the invariant state vector $|\psi(\tau)\rangle$, as follows:

$M|\psi(\tau)\rangle=\lim _{\nu \rightarrow 0} \int d u_{\tau} \psi_{\bar{U}}\left(u_{\tau}, \tau\right)\left|u_{\tau}\right\rangle=\left|U_{L}(\tau)\right\rangle$

According to relation (5.18), measurement reduces the invariant state vector $|\psi(\tau)\rangle$ into a single observable basis element $\left|u_{\tau}\right\rangle=\left|U_{L}(\tau)\right\rangle$ corresponding to a definite interface position $U_{L}(\tau)$ and consequently, there is no more any wavefunction. 
Measurement is thus, a two-step process consisting of a first local act on the projected non-local noumenal field (represented by the invariant wavefunction $\psi_{\bar{U}}\left(u_{\tau}, \tau\right)$ ) and a second non-local action of the projected non-local noumenal field via noumenal space on the corresponding noumenal particle.

Before measurement, all shadow-positions related to the arguments $\left(u_{\tau}, \tau\right)$ of the invariant wave function $\psi_{\bar{U}}\left(u_{\tau}, \tau\right)$, are possible outcomes but with different probabilities depending on the distance of each shadow-position $\left(u_{\tau}, \tau\right)$ from the noumenal position $\bar{U}=\left(U_{v}, v\right)$ of the particle. Indeed, the wavefunction depends on the distribution of distances of its different arguments from the noumenal particle and should thus, be related to the probability distribution of the particle's position outcome. The projected position $U(\tau)$ represents the shadow position on the interface spacetime having the minimal distance from the noumenal particle and is thus associated with the highest probability amplitude which decreases as we recede from the current projected position $U(\tau)$. Therefore, when a measurement is to be conducted on the noumenal particle, the likelihood is maximal that it would pop out in the neighborhood of the latest projected position.

Moreover, once the particle is in the phenomenal interface slice $S_{\tau}$ and as long as it remains there, the non-local projected noumenal field and hence, its associated wavefunction exists no more, the particle behaves then as a classical corpuscular.

Therefore, the particle has always a definite position which is in noumenal space before measurement and in phenomenal spacetime after measurement. The distinction between a quantum object and a classical object becomes only the location of that object being either in noumenal space or phenomenal spacetime, respectively.

\subsection{Plurality of particles}

More generally, a set of noumenal particles located at noumenal positions $\bar{U}=\left(\bar{U}_{1}, \bar{U}_{2}, \ldots \bar{U}_{k}\right)=$ $\left(\left(U_{1}, v_{1}\right), \ldots,\left(U_{k}, v_{k}\right)\right)$, creates a projected non-local noumenal field $\varphi_{\bar{U}}$ at each point $\left(u_{\tau}, \tau\right)$ of the interface slice $S_{\tau}$. Let $u_{\tau 1}, u_{\tau 2}, \ldots u_{\tau k}$ be a set of arguments associated to each interface point $\mathrm{u}_{\tau}$ and corresponding to the set of noumenal positions $\bar{U}_{1}, \bar{U}_{2}, \ldots \bar{U}_{k}$, then the projected non-local noumenal field $\varphi_{\bar{U}}$ maps each set of arguments $u_{\tau 1}, u_{\tau 2}, \ldots u_{\tau k}$ into a corresponding complex number $\varphi_{\bar{U}}\left(u_{\tau 1}, u_{\tau 2}, \ldots u_{\tau k}, \tau\right)$ characterizing the projected non-local noumenal field effect at each corresponding interface point, as follows:

$\left\{\left(u_{\tau 1}, u_{\tau 2}, \ldots u_{\tau k}, \tau\right)\right\} \stackrel{\varphi_{\bar{U}}}{\longrightarrow}\left\{\varphi_{\bar{U}}\left(u_{\tau 1}, u_{\tau 2}, \ldots u_{\tau k}, \tau\right)\right\}$

where $\varphi_{\bar{U}}\left(u_{\tau 1}, u_{\tau 2}, \ldots u_{\tau k}, \tau\right)$ is the projected non-local noumenal field at the interface point $\left(\mathrm{u}_{\tau}, \tau\right)$ on the interface slice $S_{\tau}$ with respect to each individual argument of the set of arguments $u_{\tau 1}, u_{\tau 2}, \ldots u_{\tau k}$. As in the case of one particle, the projected non-local noumenal field $\varphi_{\bar{U}}\left(u_{\tau 1}, u_{\tau 2}, \ldots u_{\tau k}, \tau\right)$ generated by the plurality of noumenal particles is represented by the following invariant wavefunction:

$\varphi_{\bar{U}}\left(u_{\tau 1}, u_{\tau 2}, \ldots u_{\tau k}, \tau\right) \leftrightarrow \psi_{\bar{U}}\left(u_{\tau 1}, u_{\tau 2}, \ldots u_{\tau k}, \tau\right)$

The invariant wave function $\psi_{\bar{U}}\left(u_{\tau 1}, u_{\tau 2}, \ldots u_{\tau k}, \tau\right)$ is defined in a configuration space on $R^{3 k} \times R$ and represents the projected noumenal field on the interface slice $S_{\tau}$ with respect to the different noumenal particles thus, giving an indirect information about their different noumenal positions. All the arguments $u_{1}, \ldots, u_{k}$ are defined at the same invariant interface instant $\tau$ from the standpoint of the interface slice $S_{\tau}$. Each set of arguments $u_{1}, \ldots, u_{k}$ can be considered to represent a set of 'shadow-positions' associated to a set of 'shadow-momenta' corresponding to the set of noumenal particles which have real noumenal positions $\bar{U}_{1}, \bar{U}_{2}, \ldots \bar{U}_{k}$. The shadow-positions and associated shadow-momenta can be considered as the positions and momenta of a set of k fictious particles omnipresent all over the interface slice $S_{\tau}$. Thus, each point of the interface slice $S_{\tau}$ can be considered to correspond to a set of k shadow positions. 
Let the invariant wavefunction function $\psi_{\bar{U}}\left(u_{\tau 1}, u_{\tau 2}, \ldots u_{\tau k}, \tau\right)$ be expressed in polar form in function of amplitude $R_{\bar{U}}\left(u_{\tau 1}, u_{\tau 2}, \ldots u_{\tau k}, \tau\right)$ and phase $S_{\bar{U}}\left(u_{\tau 1}, u_{\tau 2}, \ldots u_{\tau k}, \tau\right)$, as follows:

$\psi_{\bar{U}}\left(u_{\tau 1}, u_{\tau 2}, \ldots u_{\tau k}, \tau\right)=R_{\bar{U}}\left(u_{\tau 1}, u_{\tau 2}, \ldots u_{\tau k}, \tau\right) e^{i S_{\bar{U}}\left(u_{\tau 1}, u_{\tau 2}, \ldots u_{\tau k}, \tau\right)}$

As in expression (5.14), the phase $S_{\bar{U}}\left(u_{\tau 1}, u_{\tau 2}, \ldots u_{\tau k}, \tau\right)$ should inherently depend on the set of projected positions $U_{1}, U_{2}, \ldots, U_{k}$ related to a corresponding set of momenta $p_{\tau 1}, p_{\tau 2}, \ldots p_{\tau k}$. A variation in a projected position $U_{k}$ corresponds to a variation in the phase $S_{\bar{U}}\left(u_{\tau 1}, u_{\tau 2}, \ldots u_{\tau k}, \tau\right)$. On the other hand, similarly to expression (5.15), the amplitude $R_{\bar{U}}\left(u_{\tau 1}, u_{\tau 2}, \ldots u_{\tau k}, \tau\right)$ should inherently depend on the set of extradimensional space coordinates $v_{1}, v_{2}, \ldots, v_{k}$.

If the noumenal particles are correlated such that they are interlinked by the same non-local noumenal field $\varphi_{\bar{U}}$, then a variation in any noumenal position $\delta \bar{U}_{j}$ generates a variation in the projected non-local noumenal field $\delta \varphi_{\bar{U}}$ which in its turn, induces variations at all noumenal positions $\bar{U}_{1}, \bar{U}_{2}, \ldots \bar{U}_{k}$. Moreover, in that case the invariant wavefunction $\psi_{\bar{U}}\left(u_{\tau 1}, u_{\tau 2}, \ldots u_{\tau k}, \tau\right)$ cannot be factorized.

However, if the noumenal particles are not correlated, then the projected non-local noumenal field $\varphi_{\bar{U}}$ can be decomposed into a set of independent individual applications $\varphi_{\bar{U}_{1}}, \varphi_{\bar{U}_{2}}, \ldots, \varphi_{\bar{U}_{k}}$ that respectively maps each set of arguments $u_{\tau 1}, u_{\tau 2}, \ldots u_{\tau k}$ of each interface point $u_{\tau}$ into a corresponding set of complex numbers $\left(\varphi_{\bar{U}_{1}}\left(u_{\tau 1}\right), \varphi_{\bar{U}_{2}}\left(u_{\tau 2}\right), \ldots \varphi_{\bar{U}_{1}}\left(u_{\tau k}\right)\right)$ :

$\left\{\left(u_{\tau 1}, \ldots u_{\tau k}, \tau\right)\right\} \stackrel{\varphi_{\bar{U}}}{\longrightarrow}\left\{\varphi_{\bar{U}}\left(u_{\tau 1}, \ldots u_{\tau k}, \tau\right)\right\}=\left\{\left(\varphi_{\bar{U}_{1}}\left(u_{\tau 1}\right), \ldots \varphi_{\bar{U}_{k}}\left(u_{\tau k}\right), \tau\right)\right\}$

In that case, a variation in any noumenal position $\delta \bar{U}_{j}$ generates a variation only in its corresponding projected non-local noumenal field $\delta \varphi_{\bar{U}_{j}}$ which has no effect on all other noumenal positions. Each individual non-local noumenal field $\varphi_{\bar{U}_{j}}$ is related to a corresponding individual invariant wavefunction $\psi_{\bar{U}_{j}}\left(u_{\tau j}\right)$ and thus, the resultant invariant wavefunction $\psi_{\bar{U}}\left(u_{\tau 1}, u_{\tau 2}, \ldots u_{\tau k}, \tau\right)$ can be factorized into a corresponding product of independent individual invariant wavefunctions:

$\psi_{\bar{U}}\left(u_{\tau 1}, u_{\tau 2}, \ldots u_{\tau k}, \tau\right)=\psi_{\bar{U}_{1}}\left(u_{\tau 1}, \tau\right) \psi_{\bar{U}_{2}}\left(u_{\tau 2}, \tau\right) \ldots \psi_{\bar{U}_{k}}\left(u_{\tau k}, \tau\right)$

The measurement relation (5.17) can be easily generalized for numerous particles, such that a measurement conducted on one noumenal particle associated to the noumenal position $\bar{U}_{j}=\left(U_{j}, v_{j}\right)$ is defined as follows:

$M \psi_{\bar{U}}\left(u_{\tau 1}, u_{\tau 2}, \ldots u_{\tau k}, \tau\right)=\lim _{v_{j} \rightarrow 0} \psi_{\bar{U}}\left(u_{\tau 1}, u_{\tau 2}, \ldots u_{\tau k}, \tau\right)$

If the noumenal particles are not correlated, then the position of the noumenal particle under the process of measurement will tend towards the latest projected position $U_{L j}(\tau)$ and the corresponding elementary wavefunction $\psi_{\bar{U}_{j}}\left(u_{\tau j}, \tau\right)$ exists no more while all other elementary wavefunctions $\psi_{\bar{U}_{1}}\left(u_{\tau 1}, \tau\right), \cdots, \psi_{\bar{U}_{k}}\left(u_{\tau k}, \tau\right)$ are unaffected.

However, If the noumenal particles are correlated, then they form a holistic identity having a single state at any given invariant space coordinate $v$. Entangled particles are systematically kept connected to each other by the non-local noumenal field such that their invariant coordinate $v$ has constantly the same value. If the coordinate $v$ changes for one noumenal particle, it changes systematically for all others. In other words, correlated noumenal particles should always have a common extradimensional space coordinate $v$. Thus, if a particle is measured at one end, its coordinate $v$ becomes equal to zero and systematically via the non-local noumenal filed, the coordinate $v$ for all other entangled particles becomes also equal to zero and hence, all entangled particles pop-up simultaneously on the same phenomenal interface slice $S_{\tau}$. However, once the particles are localized on the interface slice $S_{\tau}$, there 
is no more any non-local projected field or wavefunction and thus, the particles are not entangled any more though there may still exist a deterministic but local relation between them.

Finally, proposition 1, also applies to a plurality of particles. Indeed, the noumenal positions of noumenal particles can be specified from the perspective of the interface spacetime, by an indirect noumenal state $\Omega_{\bar{U}}(\tau)$ composed of the invariant wavefunction $\psi_{\bar{U}}\left(u_{\tau 1}, u_{\tau 2}, \ldots u_{\tau k}, \tau\right)$ in association to a vector $U(\tau)$ defining the projected positions $\left(U_{1}, U_{2}, \ldots, U_{k}, \tau\right)$ of the noumenal particles on the interface slice $S_{\tau}$ at an invariant instant $\tau$ as well as the vector $V$ of the corresponding extradimensional space coordinates $v_{1}, v_{2}, \ldots, v_{k}$ :

$\Omega_{\bar{U}}(\tau)=\left(\psi_{\bar{U}}(\tau), U(\tau), \mathrm{V}, \tau\right)=\left(\psi_{\bar{U}}\left(u_{\tau 1}, \ldots, u_{\tau k}, \tau\right),\left(U_{1}, U_{2}, \ldots, U_{k}, \tau\right),\left(v_{1}, v_{2}, \ldots, v_{k}, \tau\right)\right)$

\section{Approximation of the interface spacetime and the invariant state vector}

The invariant state vector $|\psi(\tau)\rangle$ of equation (3.2) can also be expressed as a superposition of standard position elements $\left|x_{\tau}\right\rangle$ labelled by the continuous position variables $x_{\tau}$ of the phenomenal standard spacetime $P(x, t)$. As noted in section 2, there exists a bijective parametrization of the interface slice $\mathrm{S}_{\tau}$, such that each interface point $\left(\mathrm{u}_{\tau}, \tau\right)$ defined in the interface frame of reference $\left(0 ; \mathrm{R}_{\mathrm{u}}, \mathrm{S}_{\tau}\right)$ can be mapped into a corresponding standard point $\left(x_{\tau}, t_{\tau}\right)$ in the orthonormal frame of reference $(0 ; \mathrm{x}, \mathrm{t})$ and vice-versa. Indeed, according to expression (2.14), the points $u_{\tau}$ belonging to the slice $S_{\tau}$ can be parametrized by the standard space coordinates $x$ as follows:

$u_{\tau}(x)=\operatorname{arcsinh}\left(x_{\tau} / \tau\right)$

Thus, the invariant state vector $|\psi(\tau)\rangle$ can be expressed as follows:

$|\psi(\tau)\rangle=\int d u_{\tau} \psi_{\bar{U}}\left(u_{\tau}, \tau\right)\left|u_{\tau}\right\rangle=\int d x_{\tau} u_{\tau}^{\prime} \psi_{\bar{U}}\left(u_{\tau}(x), \tau\right)\left|u_{\tau}\right\rangle$

By differentiating $u_{\tau}(x)$ with respect to $x$ and as time $\tau$ is invariant within each slice $\mathrm{S}_{\tau}$, we get:

$u_{\tau}^{\prime}=\frac{d x_{\tau}}{\tau \sqrt{1+\left(x_{\tau} / \tau\right)^{2}}}$

Introducing $u_{\tau}^{\prime}$ into equation (6.2), the invariant state vector $|\psi(\tau)\rangle$ becomes:

$|\psi(\tau)\rangle=\int d x_{\tau} \frac{1}{\sqrt{\tau^{2}+x_{\tau}^{2}}} \psi_{\bar{U}}\left(\operatorname{arcsinh}\left(x_{\tau} / \tau\right), \tau\right)\left|x_{\tau}\right\rangle$

Let $\psi_{x}\left(\tau, x_{\tau}\right)$ be expressed as follows:

$\psi_{x}\left(\tau, x_{\tau}\right)=\frac{A}{\sqrt{\tau^{2}+x_{\tau}^{2}}} \psi_{\bar{U}}\left(\operatorname{arcsinh}\left(x_{\tau} / \tau\right), \tau\right)$

where $\mathrm{A}$ is a normalisation constant, then, the invariant state vector $|\psi(\tau)\rangle$ can be unambiguously expressed as a superposition of standard position elements $\left|x_{\tau}\right\rangle$, as follows:

$|\psi(\tau)\rangle=\int d x_{\tau} \psi_{\bar{U}}\left(x_{\tau}, \tau\right)\left|x_{\tau}\right\rangle$

where $\psi_{\bar{U}}\left(x_{\tau}, \tau\right)$ is a hybrid invariant wavefunction depending on invariant time $\tau$ and standard spatial positions $x_{\tau}$ and where for simplicity, we kept the same symbol ' $\psi_{\bar{U}}$ ' for $\psi_{\bar{U}}\left(u_{\tau}, \tau\right)$ and $\psi_{\bar{U}}\left(x_{\tau}, \tau\right)$.

However, we note that in the non-relativistic limit (i.e. $/ t \ll 1$ ) each slice $S_{\tau}$ becomes a 'quasi-interfaceline' $L_{t}$ parallel to the x-axis. Thus, the invariant time $\tau$ can be approximated by the standard time $t$. Indeed, expression (2.7) can be expressed as follows:

$\tau^{2}=t^{2}\left(1-x^{2} / t^{2}\right) \approx t^{2}$ 
Thus, $\tau \approx t$, and the phenomenal interface spacetime becomes a set of quasi-interface-lines $L_{t}$, defined as follows:

$\mathcal{J}_{t}=\left\{(x, t) \in L_{t}: x \in R, t \in R,\{|x\rangle\}\right.$ is the base that spans $\left.\mathcal{H}\right\}$

The noumenal space becomes a 'quasi-noumenal space' where the noumenal slice $S_{v}$ is approximated by a 'quasi-noumenal-line' $L_{v}$, defined as follows:

$\mathcal{N}_{t}=\left\{\bar{x}=\left(x_{v}, v\right) \in L_{v}: x_{v}, v \in R\right.$ and $\left.v \neq 0\right\}=\left\{L_{v}\right\}$

The global space becomes a 'quasi-global space' $\mathcal{G}_{t}$ defined as the union of the quasi-noumenal space $\mathcal{N}_{t}$ and the quasi-interface spacetime $\mathcal{J}_{t}$.

Therefore, in the non-relativistic limit (i.e. $x / t \ll 1$ ), the invariant time $\tau$ can be replaced by the standard time $t$ in equation (6.6) and the invariant state vector $|\psi(\tau)\rangle$ becomes a standard state vector $|\psi(t)\rangle$ in function of standard temporal and spatial coordinates $(x, t)$ :

$|\psi(t)\rangle=\int d x \psi_{\bar{U}}(x, t)|x\rangle$

The approximated wavefunction $\psi_{\bar{U}}(x, t)$ represents the state of the noumenal physical system from the viewpoint of the quasi-interface-line $L_{t}$. For simplicity, the index ' $\bar{U}$ ' is dropped from $\psi_{\bar{U}}(x, t)$ which shall be noted in the standard manner $\psi(x, t)$ by keeping in mind that the noumenal position is hidden or implicitly defined in the expression of the wavefunction.

Moreover, the invariant time coordinate $\tau$ in expression (5.13) can be approximated by the standard time coordinate $t$, leading to the standard unitary evolution of Schrodinger equation:

$|\psi(t+\delta t)\rangle=\mathcal{U}(\delta t)|\psi(t)\rangle=\exp (-i H \delta t / \hbar)|\psi(t)\rangle$

However, the above equation should be regarded as a hybrid equation of evolution where the standard time coordinate $t$ should be considered as quasi-invariant.

\section{De Broglie Bohm theory in the context of the quasi-global space}

In this section we consider the de-Broglie-Bohm theory in the formalism of the quasi-global space defined in section 6 . For simplicity, we consider a two-dimensional quasi-noumenal space composed of a set of points $\{(x, t)\}$ where $\mathrm{u}_{\tau}$ is replaced by $x$ and the invariant time $\tau$ is replaced by standard time $t$, though considered in the non-relativistic limit to be quasi-invariant. The quasi-noumenal position of the noumenal particle is noted $\bar{X}=(X, v)$, its projection is noted $X(t)$, and the non-local quasi-noumenal field is noted $\varphi_{\bar{X}}(x, t)$.

\subsection{Three rules related to the indirect noumenal state}

As outlined in section 5 (proposition 2), from the standpoint of any quasi-interface point $(x, t)$, the state of a noumenal particle located at a quasi-noumenal position $\bar{X}$ can be specified by an indirect noumenal state $\Omega_{\bar{X}}(t)$. This indirect noumenal state $\Omega_{\bar{X}}(t)$ is characterised by a wavefunction $\psi(x, t)$ and the associated projected position $X(t)$ of the noumenal particle on the quasi-interface-line $L_{t}$ as well as the corresponding extradimensional space coordinate $v$, at a quasi-invariant instant $t$ :

$$
\Omega_{\bar{X}}(t)=(\psi(x, t), \mathrm{X}(\mathrm{t}), v, t)
$$

Let the wavefunction $\psi(x, t)$ be expressed in polar form in function of amplitude $R(x, t)$ and phase $S(x, t)$, as follows:

$\psi(x, t)=R(x, t) e^{i S(x, t)}$ 
Then, the evolution of the noumenal particle obeys the following three rules:

1.The wave function $\psi(x, t)$ is governed by Schrodinger equation:

$i \hbar \frac{\partial \psi}{\partial t}=H \psi$

2. The evolution of the projected position $X(t)$ on the quasi-interface-line $\mathrm{L}_{t}$ obeys de Broglie-Bohm law:

$\frac{d}{d t} X(t)=\frac{\hbar}{m} \frac{\partial S(x, t)}{\partial x}$

3. Measurement is the limit of the wavefunction $\psi(x, t)$ as the interdimensional coordinate $v$ tends to zero, the limit being the latest projected position $X_{L}(\mathrm{t})$ :

$M \psi(x, t)=\lim _{v \rightarrow 0} \psi(x, t)=X_{L}(\mathrm{t})$

It is to be noted that in the approximation of the quasi-global space, the mathematical formalism is identical to that of the de Broglie-Bohm theory except that here, $X(t)$ is a shadow position that stands for the projected position of a noumenal particle.

Equation (7.4) shows that the projected position $X(t)$ and the phase $\mathrm{S}(\mathrm{x}, \mathrm{t})$ of the wave function $\psi(x, t)$ are interrelated in an action-reaction process. To determine the velocity of the projected position $X(t)$, the gradient of the phase $\mathrm{S}(\mathrm{x}, \mathrm{t})$ should be evaluated at the projected position $X(t)$ and thus, equation (7.4) becomes:

$\frac{d}{d t} X(t)=\left.\frac{\hbar}{m} \frac{\partial S(x, t)}{\partial x}\right|_{x=X(t)}$

Equation (7.3) governs a deterministic evolution of the non-local noumenal field between subsequent quasi-interface-lines while considering the indirect non-local relation between the different points of each quasi-interface-line $L_{t}$. Each point $(x, t)$ (i.e. shadow position) of the quasi-interface-line $L_{t}$ which corresponds to an argument of the wavefunction $\psi(x, t)$, relates directly to the noumenal particle and only indirectly via the noumenal particle to its neighbouring points (i.e. neighbouring shadow positions). The indirect relations between the arguments of the wavefunction take place via non-local field lines across the noumenal space and does not depend on the separation between the corresponding points on the quasi-interface-line $L_{t}$.

\subsection{Amplitude of the wavefunction}

As seen in section 5 , the density of the non-local quasi-noumenal field $\varphi_{\bar{X}}(x, t)$ decreases as the distance $d(x(t)-\bar{X})$ between the interface point $x(t)$ and the noumenal position $\bar{X}$ increases. The amplitudesquare $R^{2}(x)$ can thus be represented by a uniform distribution of a real-valued random variable characterized by the position $x(t)$ on the quasi-interface-line $L_{t}$ and the projected position $X(t)$ as the median, knowing that $X(t)$ is the nearest to the noumenal position $\bar{X}$.

Moreover, by supposing the non-local noumenal field to be composed of homogeneous and isotropic field lines stemming out from a noumenal particle, it is geometrically trivial that when the interdimensional coordinate $v$ decreases, the density of noumenal field lines increases at the neighbourhood of the projected position $X(t)$. In other words, when $v$ decreases, $R^{2}(x)$ becomes more peaked around $X(t)$. Conversely when $v$ increases the distribution of $R^{2}(x)$ becomes uniform or flattened. This entails that the dispersion $\sigma$ of the noumenal distribution should depend on the coordinate $v$ while preserving its order (i.e. $\sigma(v)$ should be a monotonically increasing function).

The amplitude $R(x)$ can therefore be described by a normal distribution related to the position $x$ on the quasi-interface-line $L_{t}$, as follows: 
$R(x)=\frac{1}{\left(2 \pi \sigma^{2}(v)\right)^{1 / 4}} e^{-\frac{1}{4}\left(\frac{x-X(t)}{\sigma(v)}\right)^{2}}$

The median is represented by the projected position $X(t)$ of the noumenal particle from the perspective of the quasi-interface-line $L_{t}$ at a given quasi-invariant instant $t$. The difference $x-X(t)$ indicates the distance of each point $(x, t)$ of the quasi-interface-line $L_{t}$ at the instant $t$, from the projected position $X(t)$. The density distribution $R^{2}(x)$ related to the wavefunction gives a probabilistic description of the location where the noumenal particle would most likely settle down on the quasi-interface-line $L_{t}$, the probability being larger at the interface points which are nearest to the noumenal particle and thus, to the projected position $X(t)$.

The field lines are denser (i.e. have greater number of lines) between the noumenal particle and the nearest points on the interface. However, the intensity of each link is unaffected by the distance. Indeed, in the timeless noumenal space a non-local link between two points does not depend on their separation. It is explained by Tim Maudlin in his book 'Quantum non-locality and relativity' [14] that a quantum connection between entangled particles should be unattenuated, discriminating and instantaneous.

\subsection{Rough estimate of the dispersion with respect to the interdimensional coordinate $v$}

It is known [15] that a free particle, whose initial probability density is represented by a Gaussian distribution of dispersion $\sigma$, evolves according to Schrodinger's equation such that at a later time $t$, the square of its Gaussian dispersion $\sigma(t)$ evolves as follows:

$\sigma^{2}(t)=\sigma^{2}+\left(\frac{\hbar t}{2 m \sigma}\right)^{2}$

This seems to suggest that when a particle is emitted from a source, it fades away into noumenal space becoming a noumenal particle and the interdimensional coordinate $v$ keeps increasing until measurement is conducted that makes the opposite process bringing back the particle into the phenomenal interface spacetime.

As the square of the dispersion $\sigma^{2}(t)$ increases continuously with time, the initial dispersion-square $\sigma^{2}$ can be neglected from the above equation and thus, an estimate of the dispersion $\sigma(t)$ at an instant $t$ far from the initial instant can be expressed as follows:

$$
\sigma(t) \approx \frac{\hbar t}{2 m \sigma}
$$

As suggested in section 3, the extradimensional spatial coordinate $v$ is an invariant spatial coordinate equivalent to the invariant time coordinate $\tau$. To have a rough evaluation of the interdimensional coordinate $v$, the variable $t$ in the above expression can reasonably be replaced by $v / c$. This implies that the dispersion $\sigma(v)$ is approximately proportional to the interdimensional coordinate $v$, as follows:

$\sigma(v) \approx \frac{\hbar v}{2 m c \sigma}$

To have a very rough estimate of the interdimensional coordinate $v$, we ascribed Planck's length $l_{p}$ to the initial minimal dispersion, then expression (7.10) becomes:

$\sigma(v) \approx \frac{\hbar v}{2 m c l_{p}}$

For example, a very rough estimate of the dispersion of an electron in function of the interdimensional coordinate $v$, would be of the order:

$\sigma(v) \approx 10^{23} v$ 
The above expression (7.12) entails that the slightest change in $v$ greatly amplifies the dispersion $\sigma$ suggesting that the noumenal particle keeps 'hovering' faintly off the phenomenal interface spacetime.

\subsection{Measurement in relation to a standard state vector}

Equation (7.5) illustrates that measurement consists in bringing a noumenal particle from noumenal space into the quasi-interface-line $L_{t}$ and once the particle is on that line, it behaves as a point particle governed by classical laws and there in no more any wavefunction.

When the interdimensional coordinate $v$ tends to zero, the dispersion $\sigma(v)$ tends to zero and the normal distribution becomes a Dirac delta function:

$\lim _{\boldsymbol{v} \rightarrow 0} R(x, t)=\lim _{\sigma(\boldsymbol{v}) \rightarrow 0} R(x, t)=\lim _{\sigma(\boldsymbol{v}) \rightarrow 0}\left(\frac{1}{\left(2 \pi \sigma^{2}(\boldsymbol{v})\right)^{1 / 4}} e^{-\frac{1}{4}\left(\frac{x-X(t)}{\sigma(\boldsymbol{v})}\right)^{2}}\right) \sim \delta(x-X(t))$

The operation of measurement on the state vector $|\psi(t)\rangle$ can be described, as follows:

$M|\psi(t)\rangle=\lim _{v \rightarrow 0} \int d x \psi(t, x)|x\rangle=\lim _{\sigma(\boldsymbol{v}) \rightarrow 0} \int d x R(x, t) e^{i S(x, t)}|x\rangle$

However, as indicated by expression (7.13), $R(x, t)$ converges to $\delta(x-X(t))$ when $v$ (hence, $\sigma$ ) tends to zero. The noumenal position $\bar{X}=(X, v)$ tends to $(X, 0)$ where $X$ is equal to the latest projected position $X(t) \equiv X_{L}(t)$. Moreover, $e^{i S(x, t)}$ is a continuous bounded function, thus:

$\lim _{\nu \rightarrow 0} \int d x R(x, t) e^{i S(x, t)}|x\rangle=\int d x \delta(x-X(t)) e^{i S(x, t)}|x\rangle=e^{i S(X, t)}|X\rangle \equiv\left|x_{L}\right\rangle$

The above equation shows that the particle pops out at a definite position $x_{L} \equiv X_{L}(t)$ corresponding to the latest projected position. Indeed, the projected position may vary during the convergence of $v$ towards zero and hence, the position of the particle in the interface spacetime corresponds to the latest projected position. The measured position is represented by a unique base vector $\left|x_{L}\right\rangle$ and is thus observable.

It is to be noted that the going back and forth of the particle between noumenal space and phenomenal spacetime should not be restricted to measurement and should be considered also in many other quantum phenomena such as quantum tunnelling. In his book 'The Order of Time', Carlo Rovelli [16] considers that spacetime has a granular nature and a particle exists in a cloud of probabilities and jumps from one point to another. Here noumenal space can be considered to correspond to the cloud of probability of Rovelli and the jumps take place between noumenal space and phenomenal spacetime.

To sum up, the wave function $\psi(x, t)$ is an ontological entity representing the projection of a non-local noumenal field on the quasi-interface-line $L_{t}$ created by a noumenal particle and is thus, an indirect representation of the noumenal particle.

According to this model, when a particle is in noumenal space, it is not observable but, its projected non-local noumenal field is detectable and behaves in a wave-like manner. On the other hand, when it is in the interface spacetime, it becomes observable and behaves as a point particle.

For example, in the double-slit experiment, interference is the result of the wave (i.e. the projected nonlocal noumenal field) going through both slits though the particle being in noumenal space, goes through neither. However, the movement of the noumenal particle is non-locally affected by the interference such that when the interfering wave finally interacts with the detecting screen, the noumenal particle is brought into the interface spacetime as an impact on the screen whose position has been already configured by the interfering wave before the impact. When detectors are placed at the slits and the particle is detected (i.e. measured) at one of the slits means that the particle has popped out into the interface spacetime at the detected position and the associated wave does not exist any longer. The particle produces then an impact on the screen according to a classical trajectory. 


\section{Plurality of particles in the quasi-global space}

Let a set of two noumenal particles be at a definite position vector $\bar{X}$ :

$\bar{X}=\left(\bar{X}_{1}, \bar{X}_{2}\right)=\left(\left(X_{1}, v_{1}\right),\left(X_{2}, v_{2}\right)\right)$

The wavefunction representing the projected non-local noumenal field on the quasi-interface-line $L_{t}$ is defined in a configuration space $R^{2} \times R$, as follows:

$$
\psi\left(x_{1}, x_{2}, t\right)=R\left(x_{1}, x_{2}, t\right) e^{i S\left(x_{1}, x_{2}, t\right)}
$$

From the perspective of the quasi-interface-line $L_{t}$, the dynamics of the noumenal particles of masses $m_{1}$ and $m_{2}$ at the projected positions $X_{1} \equiv X_{1}(t)$ and $X_{2} \equiv X_{2}(t)$ are related to the phase $S\left(x_{1}, x_{2}, t\right)$ of the wave function $\psi\left(x_{1}, x_{2}, t\right)$, as follows:

$\frac{d X_{k}}{d t}=\left.\frac{\hbar}{m_{k}} \frac{\partial S\left(x_{1}, x_{2}, t\right)}{\partial x_{k}}\right|_{x_{k}=X_{k}}$

where the phase $S\left(x_{1}, x_{2}, t\right)$ is evaluated at the corresponding projected position. If the noumenal particles are correlated, then any change $\delta X_{1}$ in the projected position $X_{1}$ of the first noumenal particle changes the value of the phase $S\left(X_{1}+\delta X_{1}, X_{2}, t\right)$ which automatically changes the projected position of the second particle according to equation (1b) as explained by Bricmont [7].

According to this model, the entanglement between both particles is achieved by means of the non-local noumenal field. Similarly, any action on the projected non-local noumenal field within the interface spacetime has a non-local action on both particles.

\subsection{Bivariant normal distribution}

The amplitude $R\left(x_{1}, x_{2}, t\right)$ can be described by a bivariant normal distribution of the positions $x_{1}, x_{2}$ at a given instant $t$, on the interface spacetime, as follows:

$R\left(x_{1}, x_{2}\right)=\frac{1}{2 \pi \sigma\left(\boldsymbol{v}_{1}\right) \sigma\left(\boldsymbol{v}_{2}\right) \sqrt{1-\rho^{2}}} \exp \left(-\frac{1}{2\left(1-\rho^{2}\right)}\left[\frac{\left(x_{1}-X_{1}\right)^{2}}{\sigma^{2}\left(\boldsymbol{v}_{1}\right)}+\frac{\left(x_{2}-X_{2}\right)^{2}}{\sigma^{2}\left(\boldsymbol{v}_{2}\right)}-\frac{2 \rho\left(x_{1}-X_{1}\right)\left(x_{2}-X_{2}\right)}{\sigma\left(\boldsymbol{v}_{1}\right) \sigma\left(\boldsymbol{v}_{2}\right)}\right]\right)$

where $\rho$ is a constant representing a correlation coefficient.

The medians are represented by the projected positions $X_{1} \equiv X_{1}(t)$ and $X_{2} \equiv X_{2}(t)$ of the noumenal particles from the perspective of the quasi-interface-line $L_{t}$ at a given quasi-invariant instant $t$.

\subsection{Entangled particles}

If the particles are entangled, the wave function $\psi\left(x_{1}, x_{2}, t\right)$ does not factorise into a product of independent wavefunctions. In particular, the correlation coefficient $\rho$ in the expression (8.4) of the bivariant normal distribution is not equal to zero $(\rho \neq 0)$, and systematically as indicated in section 5.7, $v_{1}=v_{2}=v$, hence, $\sigma\left(v_{1}\right)=\sigma\left(v_{2}\right)=\sigma(v)$

The above expression (8.4) becomes:

$R\left(x_{1}, x_{2}\right)=\frac{1}{2 \pi \sigma^{2}(\boldsymbol{v}) \sqrt{1-\rho^{2}}} \exp \left(-\frac{\left(x_{1}-X_{1}\right)^{2}+\left(x_{2}-X_{2}\right)^{2}-2 \rho\left(x_{1}-X_{1}\right)\left(x_{2}-X_{2}\right)}{2 \sigma^{2}(\boldsymbol{v})\left(1-\rho^{2}\right)}\right)$

Let $x_{1}^{\prime}=x_{1}-X_{1}$ and $x_{2}^{\prime}=x_{2}-X_{2}$, then $R\left(x_{1}, x_{2}\right)$ transforms into $R\left(x_{1}^{\prime}+X_{1}, x_{2}^{\prime}+X_{2}\right)$ which we shall simply note $R\left(x_{1}^{\prime}, x_{2}^{\prime}\right)$, expressed as follows:

$R\left(x_{1}^{\prime}, x_{2}^{\prime}\right)=\frac{1}{2 \pi \sigma^{2}(v) \sqrt{1-\rho^{2}}} \exp \left(-\frac{\left(x \prime_{1}\right)^{2}+\left(x \prime_{2}\right)^{2}-2 \rho\left(x \prime_{1}\right)\left(x \prime_{2}\right)}{2 \sigma^{2}(v)\left(1-\rho^{2}\right)}\right)$ 
To consider the amplitude from the perspective of the first particle, we apply to the above equation (8.6), the following transformation:

$x_{2}^{\prime}=x_{3}^{\prime}+\rho x_{1}^{\prime}$

where $x_{3}^{\prime}$ is orthogonal to $x_{1}^{\prime}$, then,

$R\left(x_{1}^{\prime}{ }_{1}+X_{1}, x_{2}^{\prime}+X_{2}\right)$ transforms into $R\left(x_{1}^{\prime}+X_{1}, x^{\prime}{ }_{3}+\rho x^{\prime}{ }_{1}+X_{2}\right)$ which we shall simply note $\mathrm{R}\left(x_{1}^{\prime}, x_{3}^{\prime}\right)$, expressed as follows:

$\mathrm{R}\left(x_{1}^{\prime}{ }_{1}, x^{\prime}{ }_{3}\right)=\left(\frac{1}{\sqrt{2 \pi \sigma^{2}(\boldsymbol{v})}} \exp \left(\frac{-\left(x^{\prime}\right)^{2}}{2 \sigma^{2}(\boldsymbol{v})}\right)\right)\left(\frac{1}{\sqrt{2 \pi \sigma^{2}(\boldsymbol{v})\left(1-\rho^{2}\right)}} \exp \left(\frac{-\left(x \prime_{3}\right)^{2}}{2 \sigma^{2}(\boldsymbol{v})\left(1-\rho^{2}\right)}\right)\right)$

The above equation can be expressed as a product of two normal distributions $R_{1}\left(x_{1}^{\prime}\right)$ and $R_{2}\left(x^{\prime}{ }_{3}\right)$.

$\mathrm{R}\left(x_{1}^{\prime}{ }_{1}, x_{3}^{\prime}\right)=R_{1}\left(x_{1}^{\prime}\right) R_{2}\left(x_{3}^{\prime}\right)=R_{1}\left(x_{1}^{\prime}\right) R_{2}\left(x_{2}^{\prime}-\rho x_{1}^{\prime}\right)$

By reintroducing $x^{\prime}{ }_{1}=x_{1}-X_{1}$ and $x^{\prime}{ }_{2}=x_{2}-X_{2}$, into (8.9), it becomes:

$\mathrm{R}\left(x_{1}^{\prime}, x_{3}^{\prime}\right)=R_{1}\left(x_{1}-X_{1}\right) R_{2}\left(x_{2}-X_{2}-\rho\left(x_{1}-X_{1}\right)\right.$

The above expression clearly shows that when the particles are correlated (i.e. $\rho \neq 0$ ), the amplitude of the second particle depends on the coordinates of the first particle.

On the other hand, by applying the above transformations to the phase $S\left(x_{1}, x_{2}, t\right)$, it can be expressed as follows:

$S\left(x_{1}, x_{2}, t\right)=S\left(x_{1}^{\prime}+X_{1}, x_{2}^{\prime}+X_{2}, t\right)=S\left(x_{1}^{\prime}+X_{1}, x^{\prime}{ }_{3}+\rho x^{\prime}{ }_{1}+X_{2}\right)$

Thus, when a measurement is conducted on the first particle, we get:

$$
\begin{aligned}
& M=\lim _{\sigma(\boldsymbol{v}) \rightarrow 0} \iint d x_{1} d x_{2} R\left(x_{1}, x_{2}\right) e^{i S\left(x_{1}, x_{2}, t\right)}\left|x_{1}, x_{2}\right\rangle \\
& M=\lim _{\sigma(\boldsymbol{v}) \rightarrow 0} \iint d x_{1}^{\prime} d x_{3}^{\prime} R_{1}\left(x_{1}^{\prime}\right) R_{2}\left(x^{\prime}{ }_{3}\right) e^{i S\left(x_{1}+X_{1}, x_{3}+\rho x_{1}+X_{2}, t\right)}\left|x_{1}^{\prime}+X_{1}, x_{3}^{\prime}+\rho x_{1}^{\prime}+X_{2}\right\rangle \\
& M=\lim _{\sigma(\boldsymbol{v}) \rightarrow 0} \int R_{2}\left(x^{\prime}{ }_{3}\right) d x^{\prime} \int R_{1}\left(x_{1}^{\prime}\right) e^{i S\left(x \prime_{1}+X_{1}, x_{3}+\rho x_{1}+X_{2}, t\right)} d x_{1}^{\prime}\left|x_{1}^{\prime}+X_{1}, x_{3}^{\prime}+\rho x_{1}^{\prime}+X_{2}\right\rangle
\end{aligned}
$$

However, for $\sigma(\boldsymbol{v}) \rightarrow 0$; the first and second normal distributions become Dirac's delta functions:

$$
\begin{aligned}
& R_{1}\left(x_{1}^{\prime}\right) \rightarrow \delta\left(x_{1}^{\prime}-0\right) \\
& R_{2}\left(x_{3}^{\prime}\right) \rightarrow \delta\left(x_{3}^{\prime}-0\right)
\end{aligned}
$$

By introducing relations (8.15) and (8.16) into (8.14), it becomes:

$\mathrm{M}=\iint d x_{1}^{\prime} d x_{3}^{\prime} \delta\left(x_{1}^{\prime}-0\right) \delta\left(x_{3}^{\prime}-0\right) e^{i S\left(x_{1}+X_{1}, x_{3}+\rho x_{1}+X_{2}, t\right)}\left|x_{1}^{\prime}+X_{1}, x_{3}^{\prime}+\rho x_{1}^{\prime}+X_{2}\right\rangle$

Finally, relation (8.17) reduces into:

$\mathrm{M}=e^{i S\left(X_{1}, X_{2}, t\right)}\left|X_{1}, X_{2}\right\rangle$

Thus, both particles settle at the corresponding projected positions $X_{1}(\mathrm{t})$ and $X_{2}(t)$.

\subsection{Non-entangled particles}

When the particles are not correlated, the wavefunction $\psi\left(x_{1}, x_{2}, t\right)$ factorises into a product of independent wavefunctions:

$\psi\left(x_{1}, x_{2}, t\right)=R\left(x_{1}, x_{2}, t\right) e^{i S\left(x_{1}, x_{2}, t\right)}=\psi_{1}\left(x_{1}, t\right) \psi_{2}\left(x_{2}, t\right)$ 
The above relation (8.19) can also be expressed in polar form:

$\psi\left(x_{1}, x_{2}, t\right)=R\left(x_{1}, x_{2}, t\right) e^{i S\left(x_{1}, x_{2}, t\right)}=R_{1}\left(x_{1}, t\right) e^{i S_{1}\left(x_{1}, t\right)} R_{2}\left(x_{2}, t\right) e^{i S_{2}\left(x_{2}, t\right)}$

such that;

$S\left(x_{1}, x_{2}, t\right)=S_{1}\left(x_{1}, t\right)+S_{2}\left(x_{2}, t\right)$

$R\left(x_{1}, x_{2}, t\right)=R_{1}\left(x_{1}, t\right) R_{2}\left(x_{2}, t\right)$

Applying de Broglie Bohm law (8.3) to the phase $S\left(x_{1}, x_{2}, t\right)$ as expressed in relation (8.21), gives two independent equations:

$$
\begin{aligned}
& \frac{d X_{1}}{d t}=\left.\frac{\hbar}{m_{1}} \frac{\partial S_{1}\left(x_{1}, t\right)}{\partial x_{1}}\right|_{x_{1}=X_{1}} \\
& \frac{d X_{2}}{d t}=\left.\frac{\hbar}{m_{2}} \frac{\partial S_{2}\left(x_{2}, t\right)}{\partial x_{2}}\right|_{x_{2}=X_{2}}
\end{aligned}
$$

The projected position of each particle follows its own law independently from the other particle.

On the other hand, the amplitude is a product of two independent amplitudes such that when the dispersion $\sigma\left(v_{1}\right)$ of the first particle tends to zero, the other particle is not affected.

Indeed, when the particles are not entangled, then the correlation coefficient $\rho=0$, and $v_{1}$ and $v_{2}$ (hence, $\sigma\left(v_{1}\right)$ and $\sigma\left(v_{2}\right)$ ) are not related. As $\rho=0$, the amplitude $R\left(x_{1}, x_{2}, t\right)$ of the bivariant normal distribution at a given instant $t$, factorises into a product of independent amplitudes, as follows:

$R\left(x_{1}, x_{2}\right)=\left(\frac{1}{2 \pi \sigma\left(\boldsymbol{v}_{1}\right)} \exp \left(-\frac{\left(x_{1}-X_{1}\right)^{2}}{2 \sigma^{2}\left(\boldsymbol{v}_{1}\right)}\right)\right)\left(\frac{1}{\sigma\left(\boldsymbol{v}_{2}\right)} \exp \left(-\frac{\left(x_{2}-X_{2}\right)^{2}}{2 \sigma^{2}\left(\boldsymbol{v}_{2}\right)}\right)\right)=R_{1}\left(x_{1}, t\right) R_{2}\left(x_{2}, t\right)$

When a measurement is conducted on the first particle, we get:

$$
\begin{aligned}
& M=\lim _{\sigma\left(\boldsymbol{v}_{1}\right) \rightarrow 0} \iint d x_{1} d x_{2} R\left(x_{1}, x_{2}\right) e^{i S\left(x_{1}, x_{2}, t\right)}\left|x_{1} x_{2}\right\rangle \\
& M=\lim _{\sigma\left(\boldsymbol{v}_{1}\right) \rightarrow 0} \int R_{1}\left(x_{1}, t\right) e^{i S_{1}\left(x_{1}, t\right)} d x_{1} \int R_{2}\left(x_{2}, t\right) e^{i S_{2}\left(x_{2}, t\right)} d x_{2}\left|x_{1} x_{2}\right\rangle
\end{aligned}
$$

When $\sigma\left(v_{1}\right) \rightarrow 0$; the first normal distribution $R_{1}\left(x_{1}\right) \rightarrow \delta\left(x_{1}-X_{1}\right)$, thus:

$$
\begin{aligned}
& M=\int \delta\left(x_{1}-X_{1}\right) e^{i S_{1}\left(x_{1}, t\right)} \int R_{2}\left(x_{2}, t\right) e^{i S_{2}\left(x_{2}, t\right)} d x_{2}\left|x_{1} x_{2}\right\rangle \\
& M=e^{i S_{1}\left(X_{1}, t\right)}\left|X_{1}\right\rangle \int \psi_{2}\left(x_{2}, t\right) d x_{2}\left|x_{2}\right\rangle
\end{aligned}
$$

The first particle is thus observed at a position corresponding to $\left|X_{1}\right\rangle$ while the second particle continues to evolve independently.

\section{Uncertainty Principle}

Here, we should consider the location of the particle whether it is in noumenal space or in phenomenal spacetime:

1.When the particle is in noumenal space, it has in principle, a definite noumenal position $\bar{U}=(U, v)$ and thus, has no uncertainty in position. However, the momentum of the particle has no sense in a timeless noumenal space.

2.When the particle is in the phenomenal interface spacetime, it behaves as a classical point particle and has well determined position and momentum. 
3. When the particle is in noumenal space, it creates a non-local noumenal field whose projection on the interface spacetime is represented by a corresponding wavefunction. As seen in previous sections, the arguments of the wavefunction can be considered to represent shadow positions on the interface slice of a ubiquitous fictious particle presenting also shadow momenta. Spatial dispersion depends on the interdimensional coordinate $v$ and thus, the uncertainty in position depends on $v$ such that when the value of $v$ increses, the uncertainty in position becomes larger. The relation between the dispersion in shadow positions and the dispersion in shadow momenta of the fictitious particle is simply derived from Fourier transformations between position and momentum representations of the wavefunction. For a wavefunction defined in a standard one-dimensional spacetime, Fourier analysis shows that the dispersion $\sigma_{x}$ for the distribution of position $x$ and the dispersion $\sigma_{p}$ for the distribution of momenta $p$ satisfy the following well-known classical mathematical relation $[15,17]$ :

$\sigma_{x} \sigma_{p} \geq \hbar / 2$

When the interdimensional coordinate $v$ tends to zero or even becomes equal to zero, the uncertainty in position diminishes and even ceases to exist as the particle becomes localized in the phenomenal spacetime (above case 2).

In view of the above, it is clear that the uncertainty principle does not concern the physical particle itself whether it is located in noumenal space or in phenomenal spacetime. Nevertheless, it concerns the fictitious particle as represented by the interface wavefunction $\psi_{\bar{U}}\left(u_{\tau}, \tau\right)$ created by the noumenal particle. In other words, it concerns the density distribution of a physically real non-local projected noumenal field $\varphi_{\bar{U}}\left(u_{\tau}, \tau\right)$ within the phenomenal interface slice $S_{\tau}$ emanating from the noumenal particle.

\section{Conclusion}

The invariant space-time interval of special relativity is considered as an invariant time in a phenomenal interface spacetime accounting for the simultaneity in the arguments of the wavefunction. By extrapolation, a timeless noumenal space is constructed presenting a notion of an invariant space accounting for non-locality between the arguments of the wavefunction. Non-locality and simultaneity go in pair, there is indeed, no sense of non-local events between two points without the events being simultaneous.

The wavefunction is an ontological object that represents a non-local projected noumenal field within the phenomenal interface spacetime created by a particle located in the timeless noumenal space. The non-local connection between the different arguments of the wavefunction takes place within the timeless noumenal space through the intermediary of the particle located in the noumenal space and not within the phenomenal interface spacetime.

Measurement is the passage of a particle from the noumenal space into the phenomenal spacetime. The particle has always a definite position either in noumenal space or in the phenomenal spacetime. From the standpoint of the phenomenal spacetime, if the particle is in noumenal space, the non-local field behaves like a wave whereas, if it is in the phenomenal spacetime, the particle behaves like a classical point particle since there is no more any non-local field.

There are no different ontologies between quantum systems and classical systems. Instead, the demarcation between the quantum realm and the classical realm is based on the location of the physical system and not on its nature. From the perspective of phenomenal spacetime, any physical system located in noumenal space can only be described via its wavefunction according to quantum rules but when the same physical system is in the phenomenal spacetime it can then be described by the dynamical rules of classical mechanics. 


\section{References}

[1] Einstein A, Podolsky B, Rosen N; Podolsky; Rosen (1935). Can Quantum-Mechanical Description of Physical Reality Be Considered Complete? Phys. Rev. 47(10): 777-780.

[2] J. S. Bell (1964). On the Einstein-Poldolsky-Rosen paradox. Physics. 1 (3): 195-200.

[3] J. S. Bell (1964). On the problem of hidden variables in quantum mechanics. Rev. Modern Physics 38, 447-452 (1966).

[4] A. Aspect; P. Grangier \& G. Roger (1982). Experimental Realization of Einstein-Podolsky-RosenBohm Gedankenexperiment: “A New Violation of Bell's Inequalities”. Physical Review Letters. 49 (2): 91-94.

[5] L. de Broglie. Remarques sur la théorie de l'onde-pilote, C.R. Acad. Sci, Paris 233, 641-644 (1951).

[6] D. Bohm. A suggested interpretation of the quantum theory in terms of "hidden variables", Parts 1 and 2, Phys, Rev, 89, 166-193 (1952).

[7] J. Bricmont. Making Sense of Quantum Mechanics, (Springer 2016).

[8] Albert Einstein (1905) Zur Elektrodynamik bewegter Körper (On the Electrodynamics of Moving Bodies), Annalen der Physik 17: 891.

[9] T. Maudlin, Philosophy of Physics: Space and Time (Princeton University Press, Princeton, 2012).

[10] H. Minkowski (1909) Raum und Zeit, Jahresberichte der deutschen Mathematiker-Vereinigung 18, 75.

[11] E. Gourgoulhon Special Relativity in General Frames Springer 2013.

[12] Mc Taggart (1908) The Unreality of Time Mind 17 pp. 457-474

[13] S. Baron and K. Miller, An Introduction to the Philosophy of Time Polity Press 2019.

[14] T. Maudlin Quantum non-locality and relativity Wiley-Blackwell 2011.

[15] J. Binney and D. Skinner The Physics of Quantum Mechanics, Oxford 2014.

[16] C. Rovelli The Order of Time, Allen Lane 2018.

[17] J. Basdevant \& J. Dalibard Mécanique quantique (Polytechnique 2014). 